\title{
A turbulent jet in crossflow analysed with proper orthogonal decomposition
}

\author{
Meyer, Knud Erik; Pedersen, Jakob Martin; Özcan, Oktay
}

Published in:

Journal of Fluid Mechanics

Link to article, DOI:

$10.1017 /$ S0022112007006143

Publication date:

2007

Document Version

Publisher's PDF, also known as Version of record

Link back to DTU Orbit

Citation (APA):

Meyer, K. E., Pedersen, J. M., \& Özcan, O. (2007). A turbulent jet in crossflow analysed with proper orthogonal decomposition. Journal of Fluid Mechanics, 583, 199-227. https://doi.org/10.1017/S0022112007006143

\section{General rights}

Copyright and moral rights for the publications made accessible in the public portal are retained by the authors and/or other copyright owners and it is a condition of accessing publications that users recognise and abide by the legal requirements associated with these rights.

- Users may download and print one copy of any publication from the public portal for the purpose of private study or research.

- You may not further distribute the material or use it for any profit-making activity or commercial gain

- You may freely distribute the URL identifying the publication in the public portal

If you believe that this document breaches copyright please contact us providing details, and we will remove access to the work immediately and investigate your claim. 


\title{
A turbulent jet in crossflow analysed with proper orthogonal decomposition
}

\author{
KNUD ERIK MEYER ${ }^{1}$, JAKOB M. PEDERSEN ${ }^{1}$ \\ AND OKTAY ÖZCAN ${ }^{2}$ \\ ${ }^{1}$ Department of Mechanical Engineering, Technical University of Denmark, DK-2800 Kgs. \\ Lyngby, Denmark \\ ${ }^{2}$ Department of Mechanical Engineering, Yildiz Technical University, 34349, Istanbul, Turkey
}

(Received 1 March 2006 and in revised form 6 February 2007)

Detailed instantaneous velocity fields of a jet in crossflow have been measured with stereoscopic particle image velocimetry (PIV). The jet originated from a fully developed turbulent pipe flow and entered a crossflow with a turbulent boundary layer. The Reynolds number based on crossflow velocity and pipe diameter was 2400 and the jet to crossflow velocity ratios were $R=3.3$ and $R=1.3$. The experimental data have been analysed by proper orthogonal decomposition (POD). For $R=3.3$, the results in several different planes indicate that the wake vortices are the dominant dynamic flow structures and that they interact strongly with the jet core. The analysis identifies jet shear-layer vortices and finds that these vortical structures are more local and thus less dominant. For $R=1.3$, on the other hand, jet shear-layer vortices are the most dominant, while the wake vortices are much less important. For both cases, the analysis finds that the shear-layer vortices are not coupled to the dynamics of the wake vortices. Finally, the hanging vortices are identified and their contribution to the counter-rotating vortex pair (CVP) and interaction with the newly created wake vortices are described.

\section{Introduction}

The jet in crossflow is a common way of mixing two fluids. Practical examples are control of combustion by the so-called 'over fire air' in large boilers, mixing of gases before chemical reactions in e.g. air pollution control systems and designs for film cooling in gas turbines. There are numerous investigations of the jet in crossflow, and we will therefore discuss only a small selection. A review of literature on the jet in crossflow can be found in Margason (1993). A number of newer references are discussed below.

Most investigations of a jet in crossflow consider incoming jets and crossflows that have low turbulence and thin laminar boundary layers. Several visualization studies have been made on such flows, e.g. Fric \& Roshko (1994), Kelso, Lim \& Perry (1996) and Lim, New \& Luo (2001), and a number of complicated vortical flow structures have been identified. Figure 1 presents an overview of the most important structures. Several structures have a mean flow definition: the counter-rotating vortex pair (CVP) created below the trajectory of the jet, one or more horseshoe vortices found upstream of the jet exit and the hanging vortices identified by Yuan, Street \& Ferziger (1999). The hanging vortices are formed near the jet exit in the shear layer between the jet and the crossflow at the sides of the jet. There are at least two important non-stationary 




FigURE 1. Schematic description of vortical structures in the jet in crossflow near the jet exit.

flow structures. The first one is jet shear-layer vortices, formed especially along the upstream side but also on the lee-side of the jet as a result an instability similar to the Kelvin-Helmholtz instability. Other possible mechanisms such as elliptical instability and CVP destabilization are discussed in Blanchard, Brunet \& Merlen (1999) and Camussi, Guj \& Stella (2002). The second unsteady structure is upright vortices or wake vortices found as vertical vortices moving downstream in the wake of the jet. Figure 1 is similar to the model proposed by Fric \& Roshko (1994), but also includes the hanging vortices and the proposal by Lim, New \& Luo (2001) that the jet shear-layer vortices are open loops that merge into the CVP.

The jets in crossflow investigated in the visualization studies mentioned above have incoming flows with stationary distributions of vorticity concentrated in thin areas. This makes it possible to track the deformation of vorticity into vortical structures by adding a tracer at relevant parts of the inlets. However, most practical applications of jets in crossflow have turbulent inlet conditions. The turbulence implies that the inflow possesses significant unsteadiness that will make the resulting flow less regular. The turbulence also creates high mixing of inlet vorticity and this will produce less distinct vortical structures. Therefore the details of the flow can be different from flows investigated in the visualization studies that use laminar inflow conditions. The present study considers a turbulent jet exhausting into a relatively thick turbulent boundary layer and the main objective is to identify the resulting flow structures. Since visualization studies are less applicable due to turbulence, we will try to identify flow structures from planar velocity measurements.

Some results from the present experiment have already been presented in previous publications in terms of time-averaged data. Meyer, Özcan \& Westergaard (2002b) investigated the general three-dimensional structure of the mean field. Özcan, Meyer \& Larsen (2005) developed a method to estimate the full velocity gradient tensor of the mean field and used it to identify vortices. The results indicate that a secondary 
CVP is present above the primary one and rotates in the opposite direction. A similar flow structure has previously been observed by Moussa, Trischka \& Eskinazi (1977) for a jet discharging from a pipe protruding into the crossflow and by Haven \& Kurosaka (1997) for different shapes of jet exit holes. In Özcan et al. (2005) it is suggested that the secondary CVP is created from the lee-side vortex loops. An experiment with the same jet to crossflow velocity ratio, but with the crossflow being a fully developed turbulent flow in a square duct has been reported in Meyer, Özcan, Larsen, Gjelstrup \& Westergaard (2002a).

Yuan et al. (1999) performed large-eddy simulations of a flow configuration similar to the one used in the present study. The jet inlet was a fully developed pipe flow. An important difference to the present study was that the crossflow had a laminar boundary layer. Yuan et al. (1999) found that the CVP was an important flow structure originating from vortices created very near the jet exit. They also observed wake vortices and found that they are closely connected to the horseshoe vortex. Muppidi \& Mahesh (2005) studied the effect of incoming flow conditions on jet trajectories by using direct numerical simulations. Gopalan, Abraham \& Katz (2004) performed measurements for a case with both a turbulent crossflow boundary layer and a jet created from a turbulent pipe flow. However, their highest jet to crossflow velocity ratio was $R=2.5$ which is lower than the value of $R=3.3$ used in the present study. Gopalan et al. (2004) used several different techniques to identify wake vortices and found that for $R>2$ these vortices contain crossflow fluid only, at least at a distance of 0.6 jet diameters from the wall. This agrees with the findings of Fric \& Roshko (1994).

The wake vortices appear to be similar to the von Kármán vortex street found behind a cylinder in crossflow. However, as pointed out by Fric \& Roshko (1994), there are fundamental differences. For a cylinder, vorticity is created in the crossflow fluid at the cylinder wall leading to the wake vortices. For an incompressible jet in crossflow, vorticity cannot be created at the interface between the jet and crossflow. Instead the vortices are formed from vorticity coming from either the crossflow boundary layer or the jet inlet. Visualizations by Fric \& Roshko (1994) showed no jet fluid in the wake vortices and this suggested that the wake vortices were created with vorticity from the crossflow only. Observations by Smith \& Mungal (1998) suggest that some jet fluid is present in the upper part of the wake vortices at high velocity ratios $(R>10)$.

Many investigations have used velocity measurements employing pointwise techniques like hot-wire anemometry and laser Doppler velocimetry. These techniques give mean velocities and turbulence statistics at single points, but they provide little information about unsteady flow structures. As already discussed, turbulence in inlet flows makes flow visualization techniques using tracers less meaningful, and thus requires consideration of alternative methods.

Rivero, Ferré \& Giralt (2001) used a rake of hot wires which gave time-resolved velocities along one or more lines. Using Taylor's hypothesis, this can be translated into cuts through structures passing over the rake. The present study uses the stereoscopic particle image velocimetry (PIV) technique, which measures all three components of the instantaneous velocity vector in a plane. Each sample can be considered a 'snapshot' of a cut through the flow. Recently, there have been several studies of jets in crossflow using PIV, including Hasselbrink \& Mungal (2001), New, Lim \& Luo (2004) and Su \& Mungal (2004).

Using PIV snapshots to identify flow structures is not straightforward. A twodimensional cut through a three-dimensional structure can be difficult to interpret, 




FIGURE 2. Sketch of the experimental set-up.

and it is usually not possible to have sufficiently high time resolution to get several snapshots of the same structure. Also, a snapshot of a given flow structure will contain random fluctuations due to turbulence and may be a superposition of several structures. It is therefore necessary to use some kind of statistical analysis of the data to be able to reach conclusions about dynamic flow structures. A candidate for this type of analysis is the proper orthogonal decomposition (POD). Here, a series of measurements is decomposed into a number of modes which make up an orthonormal basis spanning the entire data set. Based on energy considerations, the POD captures the most energetic and hence largest structures of the flow in the first modes. Thus, if the dynamics of the flow is dominated by a few large flow structures the data can often be represented satisfactorily using only a few of the first modes. The most important modes will then reflect the dominating flow structures. In contrast to methods like conditional sampling, POD does not need any assumptions about the flow. The combination of PIV and POD has previously been reported by e.g. Bernero \& Fieldler (2000), Graftieaux, Michard \& Grosjean (2001) and Pedersen \& Meyer (2002).

In the present study a combination of stereoscopic PIV and POD is employed to investigate the flow structures. Stereoscopic PIV yields snapshots of the flow field containing all three instantaneous velocity components which facilitates POD analysis of turbulent kinetic energy in the plane considered. Measurements were made in a number of planes perpendicular to all three coordinate directions.

\section{Experimental set-up}

A sketch of the experimental set-up is shown in figure 2. The experiments were done in a low-speed wind tunnel with an 8:1 contraction ratio and a test section with width $300 \mathrm{~mm}$ and height $600 \mathrm{~mm}$. A thick turbulent boundary layer was created on a vertical acrylic flat plate located near one sidewall. The effective test section width was therefore $264 \mathrm{~mm}$. The jet discharged from a circular pipe with a diameter of $D=24 \mathrm{~mm}$ and a length of $2.5 \mathrm{~m}$. The jet axis was located $1350 \mathrm{~mm}$ downstream of the leading edge of the plate. The jet was located slightly asymmetrically at a distance of $336 \mathrm{~mm}$ from the bottom wall. The test section had optical access through top and bottom walls and through a window in the sidewall opposite the jet exit. The coordinate system is defined in figure 2: $x$ is the crossflow direction, $z$ is along the jet 

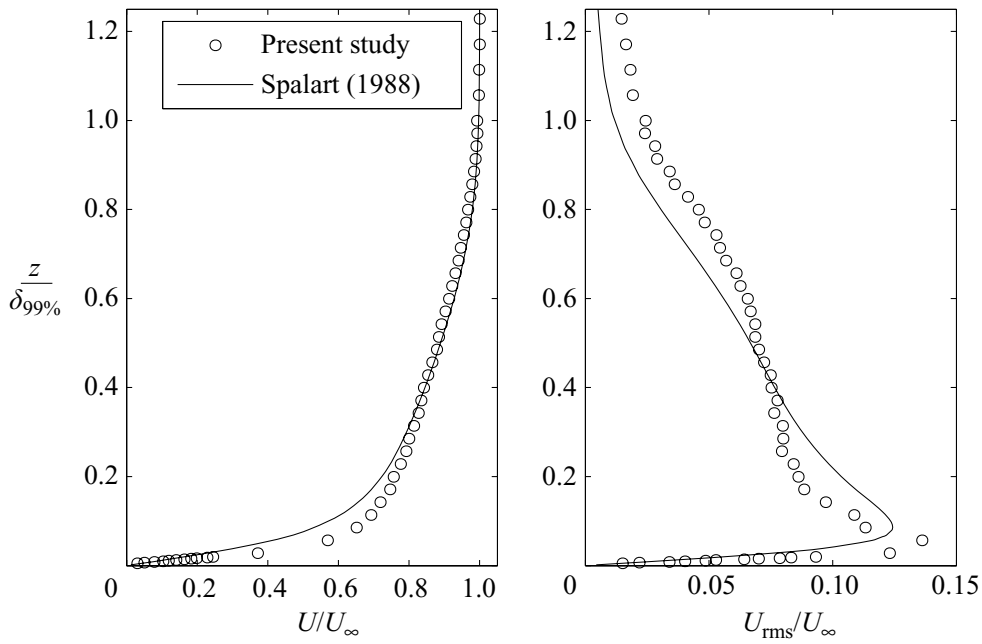

FIGURE 3. Boundary layer measured with LDA at $x / D=-3.8$ compared to DNS data by Spalart (1988).

axis, $y$ is in the spanwise direction and the origin of the coordinate system is located at the centre of the jet exit.

The experiments were conducted at a free-stream velocity (crossflow velocity) of $U_{\infty}=1.50 \pm 0.02 \mathrm{~m} \mathrm{~s}^{-1}$. The jet bulk velocity (mean velocity in the pipe) was $W_{j}=4.95 \pm 0.03 \mathrm{~m} \mathrm{~s}^{-1}$ giving a velocity ratio of $R=3.3$. A limited amount of data were also obtained for the velocity ratio of $R=1.3$ at $U_{\infty}=1.50 \mathrm{~m} \mathrm{~s}^{-1}$. The Reynolds number based on jet diameter $D$ and free-stream velocity $U_{\infty}$ was $R e=2400$ nominally. The bulk jet velocity was measured with a venturi tube that was calibrated with a reference flow meter. Static pressure measurements along the pipe showed the constant pressure gradient that is a characteristic of a fully developed flow and measured profiles of velocity and turbulence statistics near the exit showed good agreement with fully developed pipe flow, see Özcan \& Larsen (2001). Here, special consideration was given to establish and document fully developed and self-preserved turbulent incoming flow on the flat plate. This involved some manipulation of the boundary layer at the leading edge using vortex generators and a boundary layer tripping device. Figure 3 shows laser Doppler anemometry (LDA) measurements of the boundary layer upstream of the jet exit together with numerical results by Spalart (1988). $U, u_{\mathrm{rms}}$ and $\delta_{99 \%}$ are the streamwise mean velocity, root-mean-square velocity and boundary layer thickness, respectively. The agreement between measurements and Spalart's computations suggests that the incoming turbulent boundary layer can be considered to be fully developed. The boundary layer thickness was found to be $\delta_{99 \%}=70 \mathrm{~mm}$ and the displacement thickness $\delta^{*}=10.8 \mathrm{~mm}$. Additional LDA measurements from the same set-up were reported by Özcan \& Larsen (2003).

The stereoscopic PIV system consisted of two Kodak Megaplus ES 1.0 cameras (resolution of $1008 \times 1016$ ) with $60 \mathrm{~mm}$ Nikkor lenses mounted in the Scheimpflug condition. The angle between the cameras was approximately $80^{\circ}$ and the recordings used an F-number of 2.8. The light sheet of thickness $1.5 \mathrm{~mm}$ was created with a double cavity Nd-YAG laser delivering $100 \mathrm{~mJ}$ light pulses. The configuration of cameras and light sheet for measurements in constant $y$-planes is illustrated in figure 2 . For measurements in constant $x$-planes, the laser sheet optics was rotated $90^{\circ}$ around its optical axis. For measurements in $z$-constant planes, the positions of cameras and 
laser sheet optics were interchanged. Cameras and light sheet optics were mounted on the same traverse mechanism in order to accurately displace the measurement plane. Seeding consisting of $2-3 \mu \mathrm{m}$ droplets of glycerol was added to both the main flow and the jet with equal concentration based on visual evaluation of the images. The size of the seed particles was measured by an APS TSI 3320 time-of-flight spectrometer.

The images were processed with Dantec Flowmanager version 3.4 using adaptive velocity correlation and refinement steps from an initial resolution of $64 \times 64$ pixels to a final resolution of $32 \times 32$ pixels per interrogation area. Between each refinement step, the vector maps were filtered to remove spurious vectors by replacing them with a weighted-average in the neighbourhood of $3 \times 3$ vectors. $25 \%$ overlap was used between interrogation areas. A calibration target aligned with the light sheet plane was used to obtain the geometrical information required for the reconstruction of the velocity vectors. The reconstruction was performed by using a linear transformation and the calibration images recorded for five slightly displaced planes. Image maps were recorded with an acquisition rate of $0.5 \mathrm{~Hz}$, which ensured statistical independence of samples. The velocity vector maps contained typically 33 by 37 vectors. The linear dimensions of the interrogation areas varied between 1.5 and $3 \mathrm{~mm}$. The maximum spacing was slightly larger than the maximum value of the Taylor microscale, which was estimated as $2 \mathrm{~mm}$.

\section{POD analysis}

The proper orthogonal decomposition was first introduced in the context of fluid mechanics by Lumley (1967). The present analysis uses the so-called 'snapshot POD' by Sirovich (1987). Here, each instantaneous PIV measurement is considered to be a snapshot of the flow. An analysis is then performed on typically 1000 snapshots taken in the same plane. The first step is to calculate the mean velocity field, which can be considered the zeroth mode of the POD. The rest of the analysis concerns the fluctuating parts of the velocity components $\left(u_{j}^{n}, v_{j}^{n}, w_{j}^{n}\right)$ where $u, v$ and $w$ denote the fluctuating part of each of the three velocity components. Index $n$ runs through the $N$ snapshots and $j$ runs through the $M$ positions of velocity vectors in a given snapshot (i.e. $\left.u_{j}=u\left(x_{j}, y_{j}, z_{j}\right)\right)$. All fluctuating velocity components from the $N$ snapshots are arranged in a matrix $\boldsymbol{U}$ as

$$
\boldsymbol{U}=\left[\begin{array}{llll}
\boldsymbol{u}^{1} & \boldsymbol{u}^{2} \ldots & \boldsymbol{u}^{N}
\end{array}\right]=\left[\begin{array}{cccc}
u_{1}^{1} & u_{1}^{2} & \ldots & u_{1}^{N} \\
\vdots & \vdots & \vdots & \vdots \\
u_{M}^{1} & u_{M}^{2} & \ldots & u_{M}^{N} \\
v_{1}^{1} & v_{1}^{2} & \ldots & v_{1}^{N} \\
\vdots & \vdots & \vdots & \vdots \\
v_{M}^{1} & v_{M}^{2} & \ldots & v_{M}^{N} \\
w_{1}^{1} & w_{1}^{2} & \ldots & w_{1}^{N} \\
\vdots & \vdots & \vdots & \vdots \\
w_{M}^{1} & w_{M}^{2} & \ldots & w_{M}^{N}
\end{array}\right] .
$$

The autocovariance matrix is created as

$$
\widetilde{\boldsymbol{C}}=\boldsymbol{U}^{T} \boldsymbol{U}
$$


and the corresponding eigenvalue problem

$$
\widetilde{\boldsymbol{C}} \boldsymbol{A}^{i}=\lambda^{i} \boldsymbol{A}^{i}
$$

is solved. The solutions are ordered according to the size of the eigenvalues

$$
\lambda^{1}>\lambda^{2}>\cdots>\lambda^{N}=0 .
$$

The eigenvectors of (3.3) make up a basis for constructing the POD modes $\boldsymbol{\phi}^{i}$,

$$
\boldsymbol{\phi}^{i}=\frac{\sum_{n=1}^{N} A_{n}^{i} \boldsymbol{u}^{n}}{\left\|\sum_{n=1}^{N} A_{n}^{i} \boldsymbol{u}^{n}\right\|}, \quad i=1, \ldots, N,
$$

where $A_{n}^{i}$ is the $n$th component of the eigenvector corresponding to $\lambda^{i}$ from (3.3) and the discrete 2-norm is defined as

$$
\|\boldsymbol{y}\|=\sqrt{y_{1}^{2}+y_{2}^{2}+\cdots+y_{M}^{2}}
$$

Each snapshot can be expanded in a series of the POD modes with expansion coefficients $a_{i}$ for each POD mode $i$. The coefficients, also called POD coefficients, are determined by projecting the fluctuating part of the velocity field onto the POD modes

$$
\boldsymbol{a}^{n}=\boldsymbol{\Psi}^{T} \boldsymbol{u}^{n}
$$

where $\boldsymbol{\Psi}=\left[\boldsymbol{\phi}^{1} \boldsymbol{\phi}^{2} \ldots \boldsymbol{\phi}^{N}\right]$ has been introduced. The expansion of the fluctuating part of a snapshot $n$ is

$$
\boldsymbol{u}^{n}=\sum_{i=1}^{N} a_{i}^{n} \boldsymbol{\phi}^{i}=\boldsymbol{\Psi} \boldsymbol{a}^{n}
$$

It can be shown (Fukunaga 1990) that the amount of the total kinetic energy from velocity fluctuations in the snapshots that is associated with a given POD mode is proportional to the corresponding eigenvalue. The ordering of the eigenvalues and eigenvectors in (3.4) therefore ensures that the most important modes in terms of energy are the first modes. This usually means that the first modes will be associated with large-scale flow structures. If a flow has dominant flow structures, these are therefore reflected in the first POD modes and hence a given snapshot can often be reconstructed satisfactorily using only the first few modes. More details on the POD can be found in Holmes, Lumley \& Berkooz (1998) and Pedersen (2003). The snapshot POD was made using the computing language MATLAB ${ }^{\mathrm{TM}}$. Each of (3.1)-(3.7) is typically expressed as a single line of script code.

\section{Mean velocities and POD modes}

The processed PIV data will be presented as mean vector fields and POD modes found for four different cross-sections of the flow. The data from each cross-section is based on 1000 snapshots, with the exception of the $z=1.33 \mathrm{D}$ plane that is based on 658 snapshots. To investigate the dependence on the number of snapshots, the analysis has been repeated with only 200 snapshots. The results were qualitatively identical to the results based on 1000 snapshots, but there were random variations 
like slightly asymmetrical flow structures. The accuracy of the POD based on 1000 snapshots is therefore found to be satisfactory for the present analysis.

\subsection{Plotting method}

Both mean vector fields and POD modes are three-component velocity fields in a plane. The data are plotted with the in-plane components represented as velocity vectors and the out-of-plane component represented as a contour plot in greyscale. To ease comparison between different planes, the same scaling of velocity vectors and the same difference between contour levels have been used for plots of velocities and POD-modes, respectively. Coordinates are normalized with the jet inlet pipe diameter $D$. In-plane velocity vectors are scaled so that a velocity of $U_{\infty}$ corresponds to a vector length of $0.1 D$. The difference between two contour levels for the out-of-plane velocity components is $0.25 U_{\infty}$. The contour that separates positive and negative values is shown as a white dotted curve. Darker contours mark negative values and lighter contours positive values.

For the POD modes, in-plane vectors are scaled so that a unit vector has a length of $2 D$. The difference between two contour levels representing out-of-plane components is 0.01 . Again, the contour that separates positive and negative values is shown as a white dotted line with lighter contours indicating positive values. The percentage shown on POD modes is the relative value of the corresponding eigenvalue, which represents the percentage of the total kinetic energy of velocity fluctuations in the plane associated with the POD mode. Note that the length of the vectors does not have physical meaning until combined with the coefficients used in reconstruction of snapshots. However, to be able to compare the magnitude of the in-plane components with the out-of-plane components a fixed scaling is also used for plots of POD modes.

The jet trajectory can be defined by a streamline in the mean velocity field starting at the centre of the jet exit Yuan et al. (1999). This line is shown as a black dashed line on the plots of the $y=0$ plane. In the planes perpendicular to the $y=0$ plane, the intersection between this jet trajectory and the plane is marked by the symbol $\otimes$.

\subsection{The $y=0$ plane}

The best overview of the jet in crossflow is given by the $y=0$ plane, i.e. the plane aligned with the jet inlet centre axis and the free-stream flow direction. The mean velocities and the first five POD modes for this plane are shown in figure 4. The mean velocity field shows the jet entering the crossflow and being gradually deflected by the crossflow. In the wake region of the jet, the mean velocity is mainly in the $z$-direction (the jet direction), but near the jet it also has a negative $x$-component. The $y=0$ plane is a symmetry plane and the out-of-plane mean velocity component should therefore be zero. The contours seen in the mean velocity plot of figure 4 only represent small random variations due to statistical uncertainty. The velocity vectors in the first row at the jet inlet are obviously too short. The reason is that some particles made visible by the second laser pulse are inside the inlet pipe during the first pulse and they therefore do not contribute to the velocity estimate. This causes a strong velocity bias towards zero in the first row of velocity vectors at the jet inlet.

POD modes 1 and 2 shown in figure 4 are significantly stronger than the remaining modes. They each represent about $10 \%$ of the energy in velocity fluctuations whereas modes 3,4 and 5 each represent only about $3 \%$. In the $y=0$ plane, the first two modes show strong variation in the out-of-plane velocity and practically no variation in the in-plane direction. The variations are located in three parallel regions downstream of the jet trajectory. The contours of the variations are almost parallel to the jet trajectory. An interpretation of modes 1 and 2 is that the parallel regions 



Figure 4. Mean velocity and POD modes in the $y=0$ plane. See $\S 4.1$ for explanation of scaling.

of alternating positive and negative out-of-plane velocity represent vortices having different positions in each mode. In $\S 4.6$ these vortices will be identified as the wake vortices. It is interesting to note that the first mode shows out-of-plane components for $0<x / D<0.5$ near the wall, i.e. inside the jet core. This indicates that a part of the jet core interacts with the flow structures represented by modes 1 and 2 .

POD modes 3, 4 and 5 show small out-of-plane components, but have large in-plane components in the high-velocity jet region for $z / D>2$. Mode 3 should be interpreted together with the mean velocity field. If mode 3 is multiplied with a positive coefficient and added to the mean velocity field the resulting high-velocity jet region would be displaced in the downstream direction. Mode 3 therefore represents 'flapping' of the jet, i.e. changes in the degree of bending of the jet. 

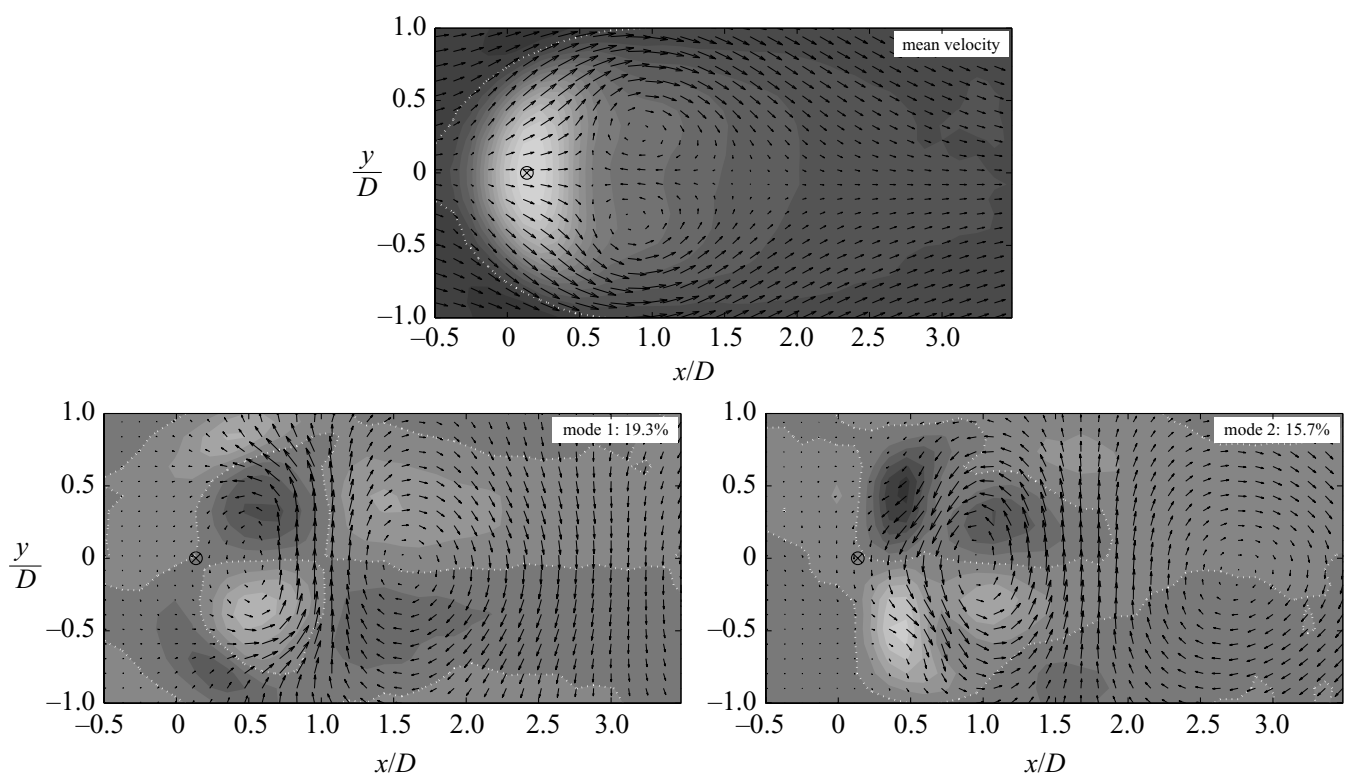

FIGURE 5. Mean velocity and POD modes in the $z=1.33 \mathrm{D}$ plane. Maximum out-of-plane mean velocity is $3.7 U_{\infty}$.

POD modes 4 and 5 clearly show in-plane vortical flow structures in the jet region. The two modes indicate almost the same structure with a shift of a quarter of a vortex width in the direction of the jet trajectory. An interpretation is that the two modes represent shear-layer vortices in the upper part of the jet. Two modes with a 'phase-shift' are needed to show that vortices are moving in the direction of the jet trajectory. Mode 5 shows regions in the wake with significant but weak variations in the out-of-plane direction. This may be seen as an indication of a connection between the vortices in modes 4 and 5 (jet shear-layer vortices) and the wake phenomena from modes 1 and 2 (wake vortices). However, modes 1-2 have no variation in the region of jet shear-layer vortices and modes 3-4 have no variation in the wake region. This suggests that the wake phenomena described by the first two modes and the jet region phenomena described by the next three modes are basically independent.

The energy associated with modes $3-5$ is significantly lower than that of modes $1-$ 2. A reason for this is that fluctuations in modes 3-5 are limited to a smaller area around the jet core. An analysis that only includes the high-velocity jet region would show modes 3-5 as the first modes and associate them with much higher relative energy. It is interesting to note that a POD analysis of the full fields that does not include the out-of-plane velocity (see Pedersen 2003) also results in the first three modes being equal to modes 3-5 in figure 4. Thus, the first two modes on the centreplane (figure 4) are almost exclusively connected to the large out-of-plane velocity fluctuations induced by the vortices that form and shed in the wake near the centreplane.

\subsection{The $z=1.33 D$ and $z=0.67 D$ planes}

Figures 5 and 6 show cross-sections through the jet core and wake. The cross-section at $z=1.33 D$ (figure 5) is at a position where the jet trajectory is beginning to bend, but before the shear-layer vortices become strong according to modes 4 and 5 in figure 4 . The plot of the mean velocities in figure 5 shows that the high-velocity 

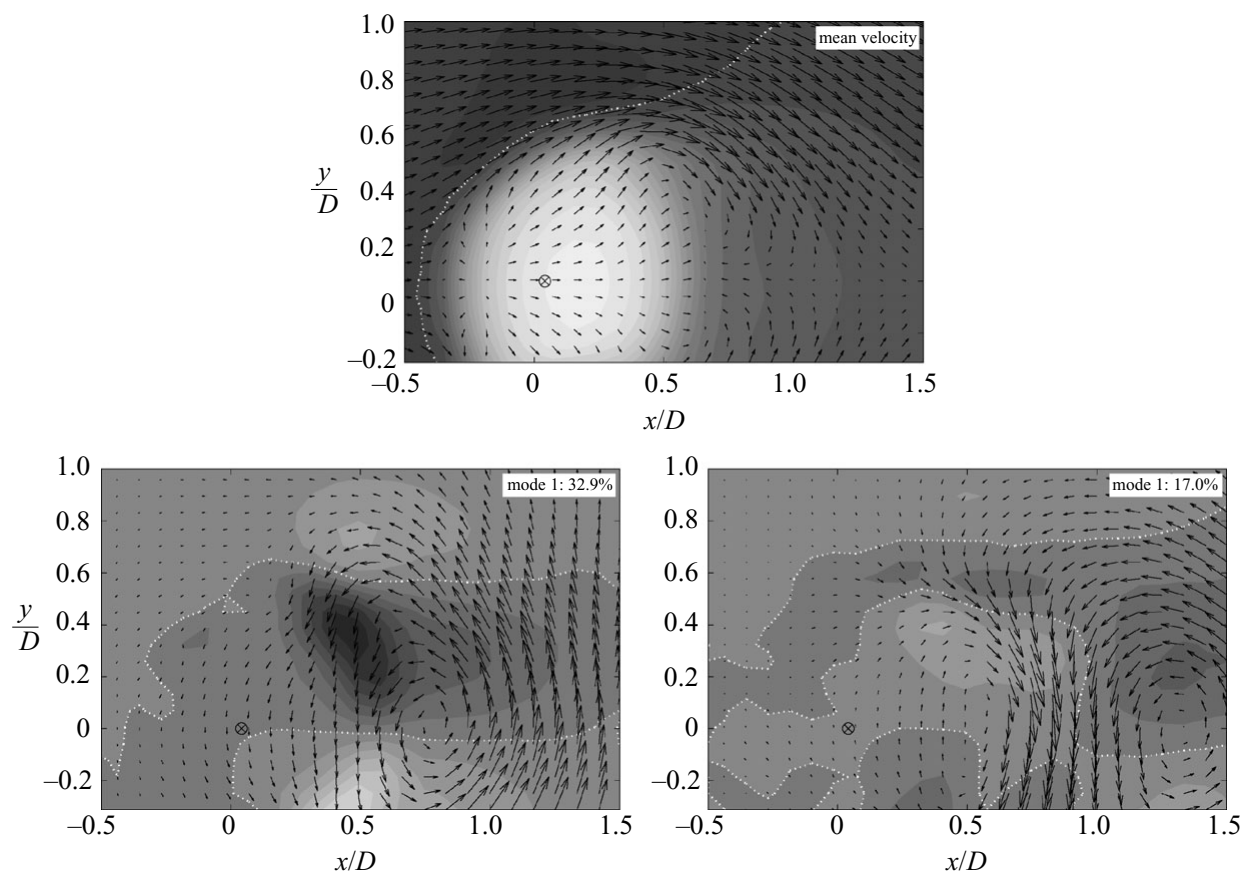

FIGURE 6. Mean velocity and POD modes in the $z=0.67 \mathrm{D}$ plane. Maximum out-of-plane mean velocity is $4.4 U_{\infty}$.

core of the jet has a semicircular shape that extends about $0.5 D$ in the $x$-direction and about $D$ in the $y$-direction. In the wake just downstream of the out-of-plane high-velocity core, the in-plane mean field shows two symmetrical vortices. All of the wake region shown has positive $w$-velocity component.

The first two POD modes of the $z=1.33 D$ plane, also shown in figure 5 , together represent $35 \%$ of the energy of velocity fluctuations. Both modes show a large inplane vortex just downstream of the high-velocity core of the jet and with centre at the $x$-axis. In the first mode, the centre of the vortex is at $x / D=1.5$. In the second mode the centre is at $x / D=1$ and a second, weaker vortex has centre at $x / D=2.7$. It is interesting to compare the first two POD modes for the $z=1.33 D$ and $y=0$ planes along the line of intersection of the two planes. For both planes, the only significant component along this line is in the $y$-direction. Mode 1 in figure 4 and mode 2 in figure 5 are in reasonable agreement: there is a significant velocity in the jet core region $(0<x / D<0.5)$ and a region with velocity in the opposite direction for $1<x / D<2$. Mode 2 in figure 4 and mode 1 in figure 5 are also in reasonable agreement: large velocities are found just behind the jet core $(0.5<x / D<1.0)$ and a large region with velocity in the opposite direction is found for $1.5<x / D<3.5$. The first two modes (with mode number interchanged) for the $y=0$ and the $z=1.33 D$ planes therefore have the same qualitative behaviour along the intersection line of the two planes.

Figure 6 shows mean velocities and POD modes for the $z=0.67 \mathrm{D}$ plane. Data in this plane were originally taken to measure the far-field flow effect and therefore the measurement field was placed asymmetrically with respect to the $x$-axis. The symmetry in the POD modes indicates that this does not affect the POD analysis. The mean velocity field shows that the out-of-plane high-velocity core of the jet is slightly deformed towards the semicircular shape seen in the $z=1.33 \mathrm{D}$ plane. Flow 

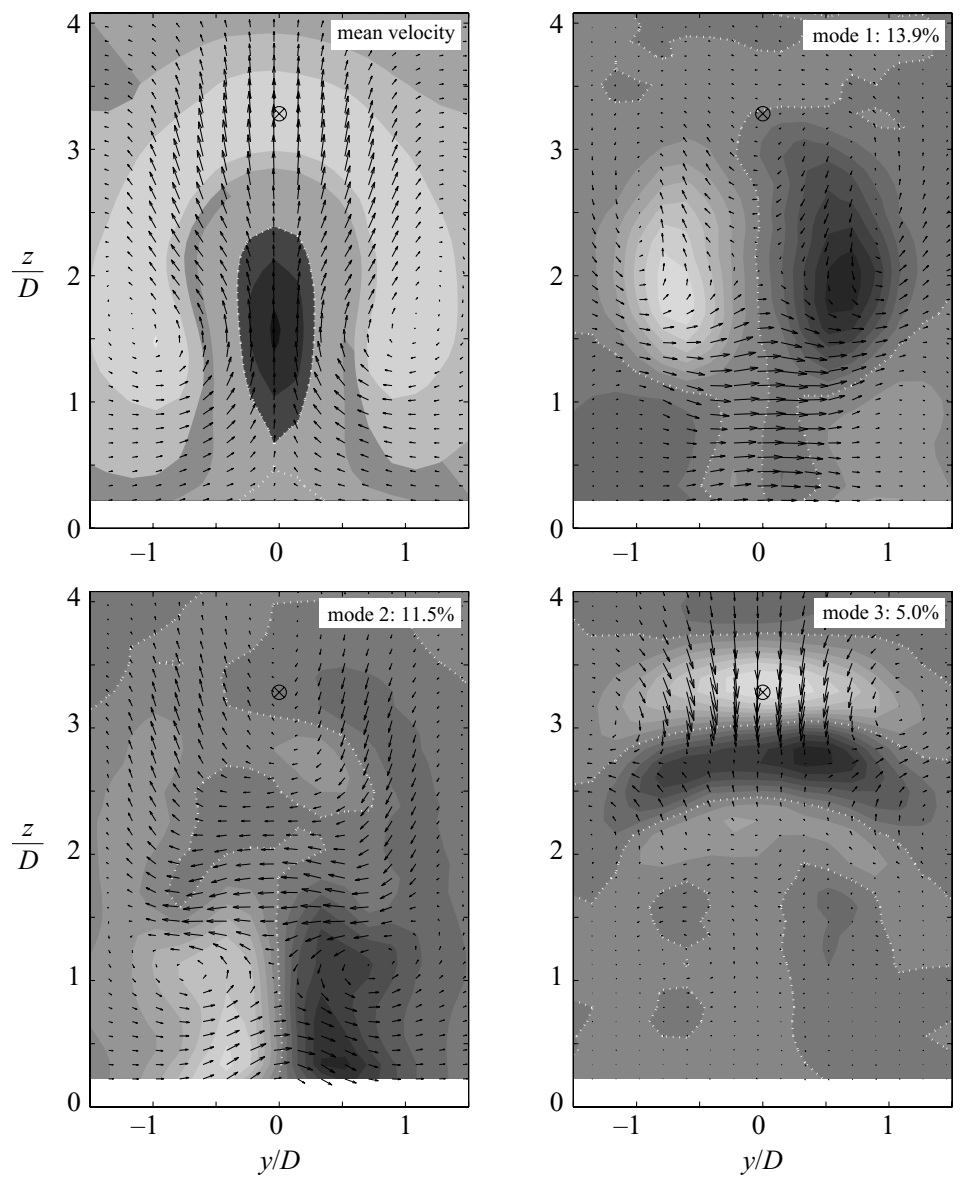

FIGURE 7. Mean velocity and POD modes in the $x=D$ plane.

recirculation can be seen in the wake of the jet, but this does not define clear vortices. The first two POD modes show the same type of in-plane vortices as found for the $z=1.33 D$ plane, but with modes 1 and 2 interchanged. The first two modes now represent $50 \%$ of the energy of the velocity fluctuations. The first mode has strong components inside the jet core. There is also reasonable agreement between the $y=0$ plane and the $z=0.67 \mathrm{D}$ plane along the intersection line for the first two modes: mode 1 has velocities in the core $(0.5<x / D<1.0)$ and in the opposite direction for $0.8<x / D<1.5$ while mode 2 has strong velocities just downstream of the jet core $(0.5<x / D<1)$ and in the opposite direction in a region further downstream.

For both the $z=1.33 \mathrm{D}$ and the $z=0.67 \mathrm{D}$ planes, the third POD mode (not shown) represents only $3 \%$ of the energy in flow fluctuations, i.e. an order of magnitude less than the first two modes together. The third and remaining modes seem to represent instantaneous variations of the flow pattern from the first two modes.

\subsection{The $x=D$ plane}

Figure 7 shows mean velocities and the first three POD modes for the $x=D$ plane. The symbol $\otimes$ at $z / D=3.3$ indicates intersection of the jet trajectory with the $x=D$ plane. The mean velocity component in the $x$-direction $U$ has a horseshoe-shaped contour. Inside the horseshoe there are negative values of $U$. The top of the horseshoe 
is at the high-velocity core of the jet (cf. figure 4), but it is interesting to note that the same magnitude of $U$ is found along the two branches of the horseshoe down to about $y / D= \pm 1$ and $z / D=1.5$. Here the in-plane vectors indicate two large vortices. These are traces of the CVP. As it will be discussed later, the CVP are taking form in this region and therefore show large variations in snapshots (e.g. see figure 15). The CVP intersect the $x=D$ plane at an angle and the lower end of the horseshoe region of high out-of-plane velocity is a part the CVP. The centre of a vortex in the CVP is therefore located closer to the $y=0$ plane than indicated by the in-plane vectors shown in figure 7 . This is consistent with the two vortices seen in the mean velocity field in figure 5. Close to the wall near $(y / D, z / D)=(0,0.25 D)$, negative out-of-plane velocities indicate that the crossflow is reversing near the wall. This flow reversal can also be seen on the mean velocity plot in figure 4 . As seen in figure 3, the crossflow boundary layer has velocities significantly below the free-stream velocity in the region $0<y / D<0.25$. The reverse flow is caused by the combined actions of the CVP and the wake vortices.

Turning to the first POD mode shown in figure 7, it is observed that for $z / D<1.5$, vectors are mainly in the positive $y$-direction. For $z / D>1.5$ vectors are mainly in the $x$-direction with the out-of-plane component being positive and negative in regions near $y / D=-0.7$ and $y / D=0.7$, respectively. The second POD mode has the opposite pattern, with vectors in the negative $y$-direction for $1.5<z / D<3$ and regions with out-of-plane variations for $z / D<1.5$. These patterns can be explained as the passage of a vortex (wake vortex) with inclined axis over the centre plane. This interpretation is supported by the fact that modes 1 and 2 in figure 7 have qualitatively the same behaviour as modes 1 and 2 in figure 4 along the intersection line of the two planes. Mode 1 has significant components near the position of the CVP. When mode 1 is added to the mean field this will cause a displacement of the CVP. An example of this will be shown in $\S 5.3$.

The third POD mode in figure 7 shows negative values of the $x$-component near $z / D=2.7$ and positive values near $z / D=3.3$, where the $z$-component shows positive values. This pattern is in reasonable agreement with mode 4 in figure 4 and this suggests that mode 3 in figure 7 is associated with the shear-layer vortices. Mode 4 of the $x=D$ plane (not shown) also has a pattern suggesting shear-layer vortices.

\subsection{POD coefficients}

For a given snapshot, the importance of the different modes can be expressed by the POD coefficients that are found by projecting the snapshot onto the POD modes as illustrated by (3.6). The relation between two modes can be shown as a scatter plot of the two coefficients found for all snapshots. Figure 8 shows such scatter plots for the coefficients of the first two modes, $a_{1}$ and $a_{2}$ for the $y=0$ and the $z=1.33 D$ planes. The scatter plot for the coefficients from the $y=0$ plane shows a circular pattern. Most points are located near a circle with radius 10 and centre at $\left(a_{1}, a_{2}\right)=(0,0)$. More than $92 \%$ of the snapshots have coefficients $\left(a_{1}, a_{2}\right)$ located outside a circle with radius 5 . The distribution of $\left(a_{1}, a_{2}\right)$ from the $z=1.33 D$ plane show a similar circular pattern, but with a slightly larger radius. Scatter plots of $\left(a_{1}, a_{2}\right)$ for the $z=0.67 D$ and $x=D$ planes (not shown) also show circular distributions. As discussed in the next section, the circular pattern indicates a strong connection between POD modes 1 and 2 .

\subsection{Interpretation of the POD analysis}

The observations related to POD modes 1 and 2 for all planes shown in the present study can be summarized as follows: (1) the modes represent an order of magnitude more energy of the velocity fluctuations than the remaining modes; (2) the modes 

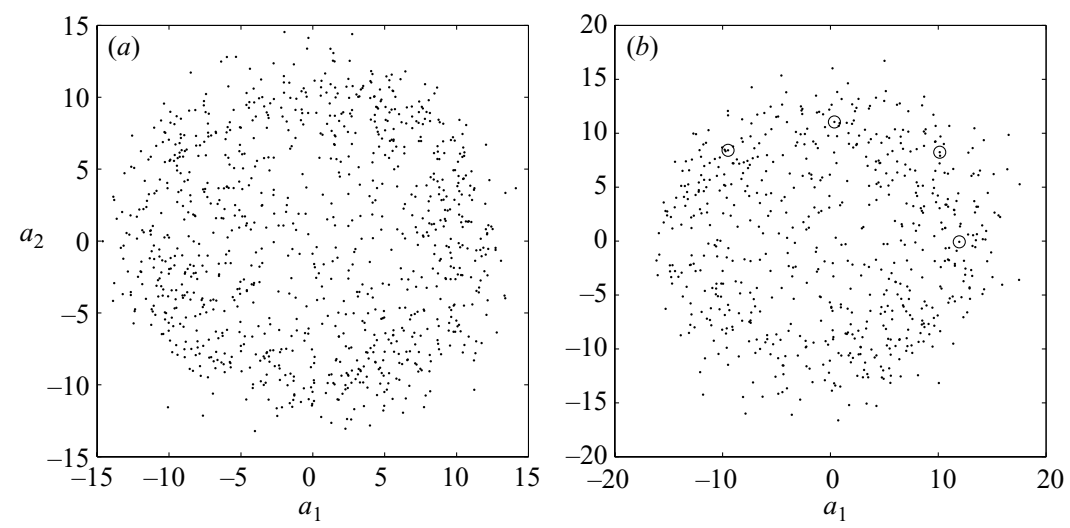

FIGURE 8. Distribution of the first and second POD mode from the $y=0$ plane $(a)$ and from the $z=1.33 D$ plane $(b)$.

are consistent with the presence of vortices in the wake of the jet; (3) mode 2 shows a flow structure displaced about a quarter of a 'vortex width' compared to mode 1; (4) the modes from the different planes agree qualitatively with each other; (5) scatter plots of the $\left(a_{1}, a_{2}\right)$ coefficients have a circular distribution.

The observations support the following interpretation: the dominant flow structure near the jet exit (in terms of energy content) seems to be vortices in the wake of the jet. These vortices have an axis almost parallel to the jet trajectory. The vortices are created at the downstream side of the jet, and they interact strongly with the jet. The vortices are convected downstream and the first two POD modes appear to describe two phases of the convective motion. The circular distribution of the $\left(a_{1}, a_{2}\right)$ coefficients suggests a cyclic variation of POD modes 1 and 2 , which is exactly what is expected if two POD modes describe different phases of a smooth process creating and convecting vortices. One would expect the $a_{1}$ and $a_{2}$ coefficients to change similarly to cosine and sine functions, respectively, in a time-resolved study of the flow. The random variations from a simple circle in figure 8 account for the influence of turbulence in terms of both local velocity fluctuations and irregularities in the creation of the vortices in the wake. Examples of the assumed cyclic variation between POD modes 1 and 2 are shown using snapshots and their reconstructions in $\S 5$. Based on the energy distribution for the POD modes, the main features of these wake vortices are fully described by the first two modes.

For the $y=0$ and the $x=D$ planes, the POD modes following the first two modes indicate the presence of the jet shear-layer vortices observed by many other authors, e.g. Fric \& Roshko (1994). As they cover much less space, these vortices represent much less energy than the wake vortices and thus are only captured by the higherorder modes. The jet shear-layer vortices cover the full high-velocity core of the jet at the point where they appear. The POD modes show either the wake vortices (modes 1-2) or the jet shear-layer vortices (modes 3-5). Thus there is no strong coupling between these two phenomena. This observation is consistent with the fact that the Strouhal number of the wake vortices (reported for example by Fric \& Roshko 1994) is different from that of the jet shear-layer vortices reported by Shapiro et al. (2006). Modes 4 and 5 in figure 4 show that the jet shear-layer vortices form along the jet trajectory about one jet diameter downstream from the the jet exit. This observation is consistent with the flow visualization results of Fric \& Roshko 

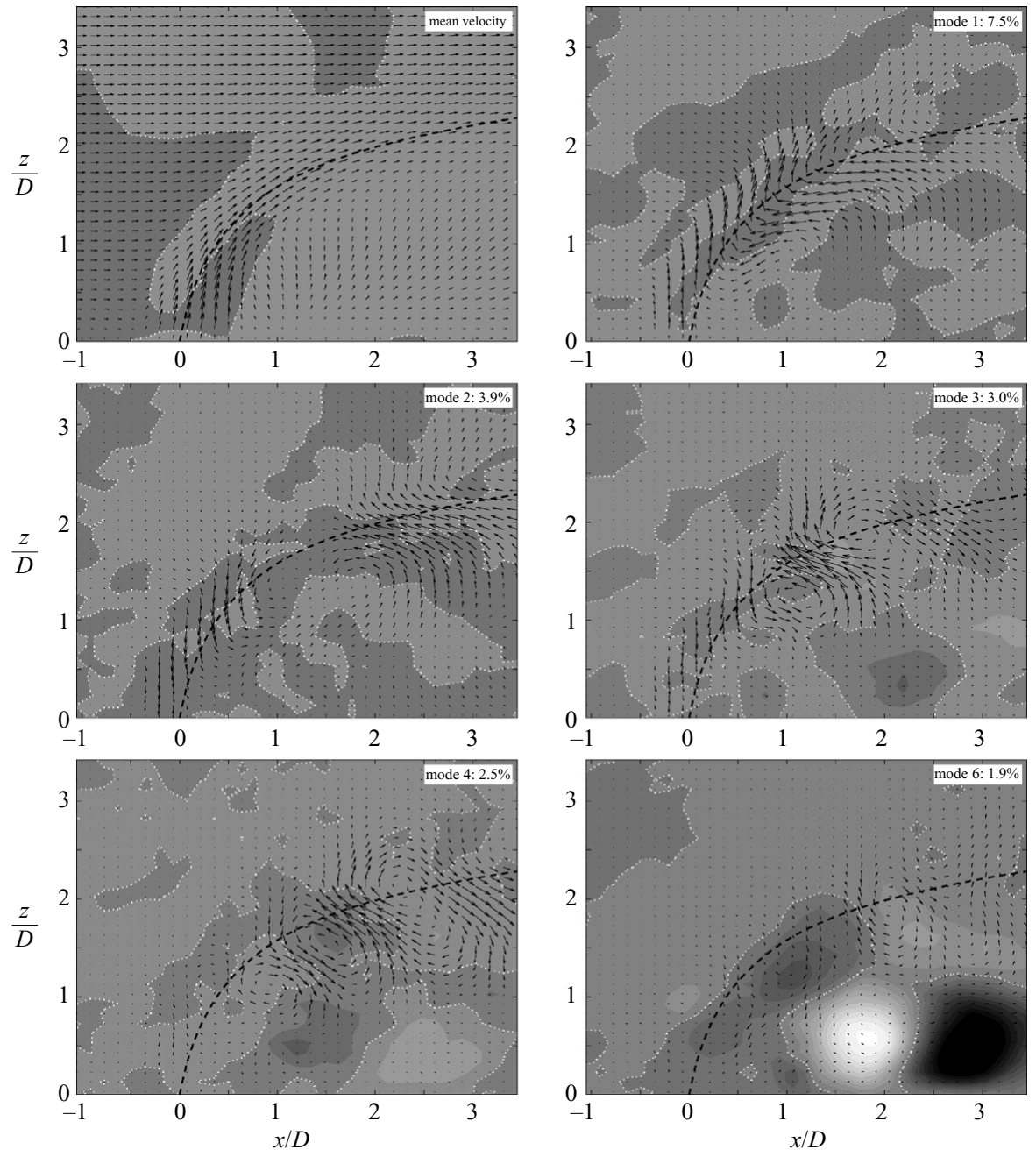

FIGURE 9. Mean velocity and POD modes in the $y=0$ plane with $R=1.3$. See $\S 4.1$ for explanation of scaling.

(1994) and Kelso et al. (1996) and points to the importance of sampling location for determining the Strouhal number of the jet shear-layer vortices.

\subsection{The $R=1.3$ jet}

A single measurement series of 1000 snapshots at the $y=0$ plane was made at a jet to crossflow velocity ratio of $R=1.3$. This measurement was performed by lowering the jet bulk velocity while keeping the crossflow velocity at $U_{\infty}=1.50 \mathrm{~m} \mathrm{~s}^{-1}$. The results in terms of the mean velocity field and the first POD modes are shown in figure 9. The mean field is similar to that of the higher jet ratio. However, the first three POD modes are quite different since they have almost no out-of-plane motion but instead resemble modes 3, 4 and 5 of the $R=3.3$ case. Modes 1 and 2 are related to a 'flapping' motion of the jet, i.e. changes of the position of the instantaneous jet trajectory, while modes 3 and 4 show shear layer vortices. Modes 3 and 4 also have a weak out-of-plane motion below the jet trajectory. This motion becomes slightly stronger in modes 5-7 (not shown), but it is not until mode 8 (shown in figure 9) 
that out-of-plane velocities become significantly stronger than in-plane velocities. The patterns of modes 8 and 9 (mode 9 not shown) are similar to the first two modes in the $R=3.3$ case and they represent the interaction of the wake vortices with the jet core. The POD analysis of the $R=1.3$ case indicates that the dominant dynamic flow structure is the shear-layer vortices and that even though wake vortices seem to exist, they only have a weak influence on the jet core and the rest of the flow.

\section{Interpretation of snapshots}

The individual PIV snapshots show both systematic and random fluctuations. They are therefore difficult to interpret. The POD modes can serve as a tool to facilitate an interpretation by focusing on those of the instantaneous fluctuations that are highly correlated and hence appear systematically. In the following discussion, snapshots are selected based on the first two POD coefficients $a_{1}$ and $a_{2}$ so that: (i) $a_{1}{ }^{2}+a_{2}{ }^{2}$ takes a value close to the average value and (ii) $a_{1} \approx 0$ or $a_{2} \approx 0$ or $\left|a_{1}\right| \approx\left|a_{2}\right|$. The second criterion corresponds to selecting a certain phase of the interaction between the two modes. For each phase, a few of the snapshots that matched the two criteria best were found and a final one was selected based on low values of the coefficients of the following modes $\left(a_{3}, a_{4}, \ldots\right)$. This approach selects snapshots that are more 'clean' than the average and hence elucidates the flow features related to the first two modes. Nevertheless, the majority of snapshots with similar values of $a_{1}$ and $a_{2}$ showed the same basic flow structures.

\subsection{Snapshots at the $z=1.33 \mathrm{D}$ plane}

Figure 10 shows four snapshots from the $z=1.33 D$ plane together with their corresponding reconstructions using the first two POD modes, i.e. using (3.7) with $N$ replaced by 2 and adding the result to the mean field. The reconstructions have been marked with the values of $a_{1}$ and $a_{2}$. The snapshots have been selected to follow the circular pattern of the distribution of $\left(a_{1}, a_{2}\right)$ seen in figure $8(b)$. Here the selected points are marked with the symbol $\otimes$. One can hypothesize that the snapshots represent different phases in time of the development of the flow. The direction of rotation in figure $8 b$ is not known, but a counter-clockwise rotation has been assumed. The first snapshot (row 1 in figure 10) is dominated by the first mode (i.e. $a_{1}$ is large and $a_{2} \approx 0$ ), the second snapshot (row 2) has $a_{1} \approx a_{2}$, the third snapshot (row 3) is dominated by the second mode $\left(a_{1} \approx 0\right)$ and the last snapshot (row 4$)$ has $a_{1} \approx-a_{2}$.

In the first snapshot and in its reconstruction (row 1 in figure 10), a clockwiserotating wake vortex is centred at $(x / D, y / D)=(1.3,0.2)$. In the second and third snapshot/reconstructions (rows 2 and 3 ) this wake vortex moves to $x / D=1.7$ and 2.5, respectively. The fourth snapshot/reconstruction (row 4) is approaching a mirror image of the first snapshot/reconstruction with a counter-clockwise vortex moving away from the jet core. This indicates a process of continuous shedding of wake vortices, and that the snapshots may indeed be ordered in phases according to the counter-clockwise circulation assumed in figure $8(b)$. When a vortical structure is seen near the jet core, an increased in-plane velocity in the jet core with an angle of $45^{\circ}$ to the $x$-axis is seen, e.g. near $(x / D, y / D)=(0.4,-0.5)$ for rows 1 and 2 . This seems to feed the vortex with jet fluid. The vortical structures that are seen near the jet core may be a paired combination of a wake vortex with the hanging vortex (the origin of the CVP). As argued in the discussion of figure 11, close proximity of the hanging vortex and the wake vortices in the $z / D=1.33$ plane makes them indistinguishable. 
(a)
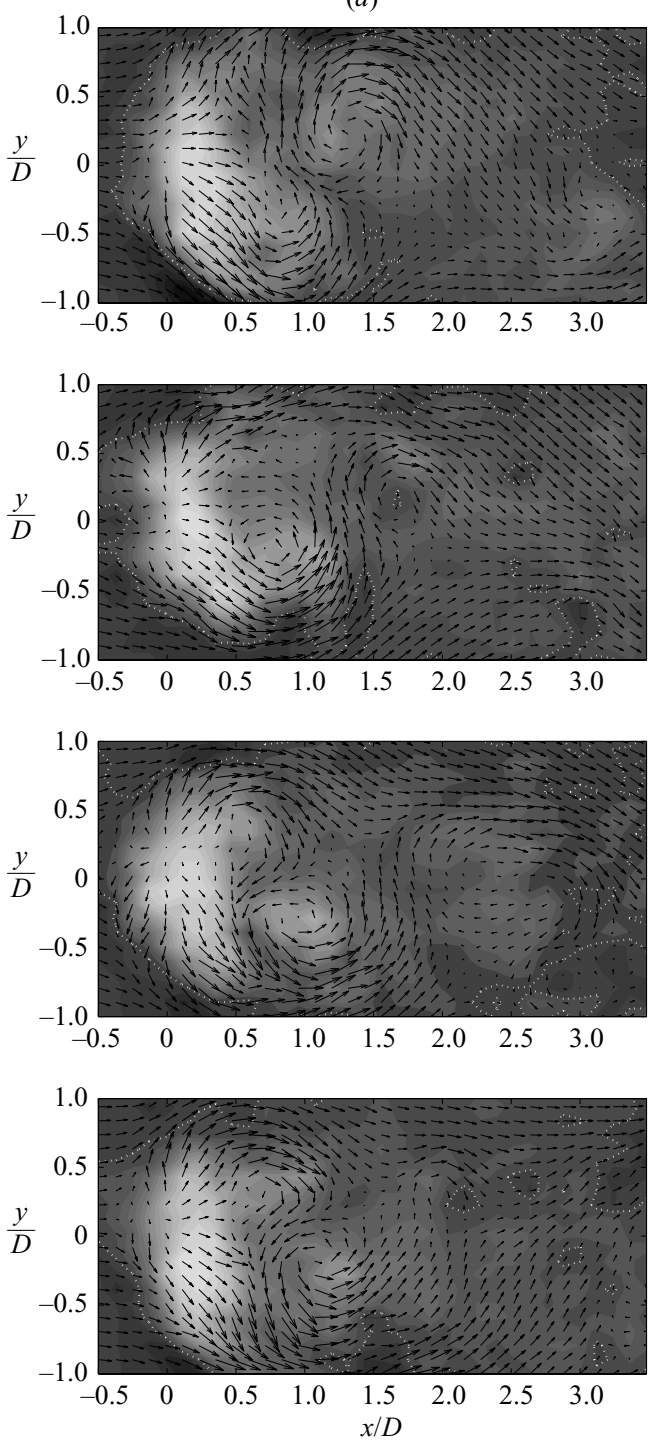

(b)


FIGURE 10. Snapshot in the $z=1.33 D$ plane $(a)$ and reconstruction $(b)$ using the first two POD modes.

The reconstructions of snapshots reveal all of the qualitative flow details found in the region in and near the jet core in the snapshots, but vortices further downstream do not appear clearly in the reconstructions. Inspection of other snapshots with similar POD coefficients (not shown) reveals that positions of downstream separated vortices vary greatly. This is why the downstream wake vortices are not captured in the first two POD modes. It implies that while the shedding of vortices seems to follow a regular pattern, their downstream transport is influenced by significant random flow variations. None of the snapshots in figure 10 shows clear vortices in the farthest downstream part. This is a typical observation. Probably the vortices are losing coherence due to diffusion of vorticity. 
Most of the vortices in the snapshots in figure 10 have a positive $w$-component in the core, which is also the case for the reconstructions. This indicates that most vortices have a tornado-like form with fluid being sucked up from the near-wall region. However, vortices with negative $w$-component in the core are also observed on a few snapshots (not shown).

It is well known that velocity vector maps are not always successful in revealing vortices. Therefore a modified version of the $Q$-criterion by Hunt, Wray \& Moin (1988) is used to detect vortical structures. The calculation of $Q$ involves a measure of the vorticity vector subtracted by a similar 'measure' of the rate-of-strain tensor. A positive value of $Q$ therefore indicates that vorticity is caused by a vortex and not by a shear layer. Since the out-of-plane velocity gradients are not known, the modification used for $Q$ is based on only in-plane velocity components and gradients. This clearly favours identification of vortices with axes perpendicular to the plane examined and it is considered to be a useful approximation for the present $z$-constant planes where in-plane gradients are much larger than out-of-plane gradients. Other criteria for detection of vortices also exist and a test has been done with the 'swirling strength' as suggested for use on two-dimensional PIV fields by Adrian, Christensen $\&$ Liu (2000). Such test plots gave almost exactly the same results as plots using the modified $Q$-criterion, which has therefore been used.

The results presented in figure 11 for the same snapshots as appear in figure 10 show values of $Q$ as greyscale contours with darker colour indicating higher values. Regions with a negative value of $Q$ have no contours (i.e. are white). The coloured regions therefore indicate the positions and extent of vortices. The same scale is used in all plots to facilitate comparison of the relative strength of the vortices. To demonstrate that a high value of the modified $Q$-criterion does identify vortices not easily seen in snapshots, we have plotted two selected snapshots with a Galilean transformation in figure 12 . Here, plot $(a)$ is from the $z=1.33 D$ plane and plot $(b)$ is from the $z=0.67 D$ plane (to be discussed in $\S 5.2$ ). In each plot the approximate centre velocity of the most upstream peak in $Q$ has been subtracted from all in-plane velocities. This creates a frame of reference that follows the flow in the possible vortex. In both cases, the Galilean transformation reveals a clear circular pattern of velocity vectors and thus a vortex - even though a vortex cannot be identified from the velocity vectors in the fixed reference view used in the original plots.

At first sight, figure 11 only reveal the vortices already discussed. On some plots in figure 11 (both vortices in row 1, lower vortex in row 2) there are two local maxima of the modified $Q$-criterion in some of the identified vortices. We will assume that this is the hanging vortex seen together with a newly created wake vortex, and that the two vortices are interacting, possibly merging.

\subsection{Snapshots at the $z=0.67 \mathrm{D}$ plane}

Figure 13 shows three selected snapshots together with their corresponding reconstructions using the first two POD modes. The same snapshots and reconstructions are also shown in figure 14 using the modified $Q$-criterion as in figure 11 .

In figure 13, the first snapshot (row 1) is dominated by the first POD mode, the second snapshot (row 2) is dominated by the second POD mode and the last snapshot (row 3 ) has $a_{1} \approx-a_{2}$. In the first snapshot (row 1), a wake vortex is visible near the downstream edge of the jet core around $(x / D, y / D)=(0.8,-0.1)$. In the lower part (towards negative $y$ ) of the jet core there is a flow with an angle of $-45^{\circ}$ with the $x$-axis. In the second snapshot (row 2), a similar vortex is seen quite a distance 
(a)
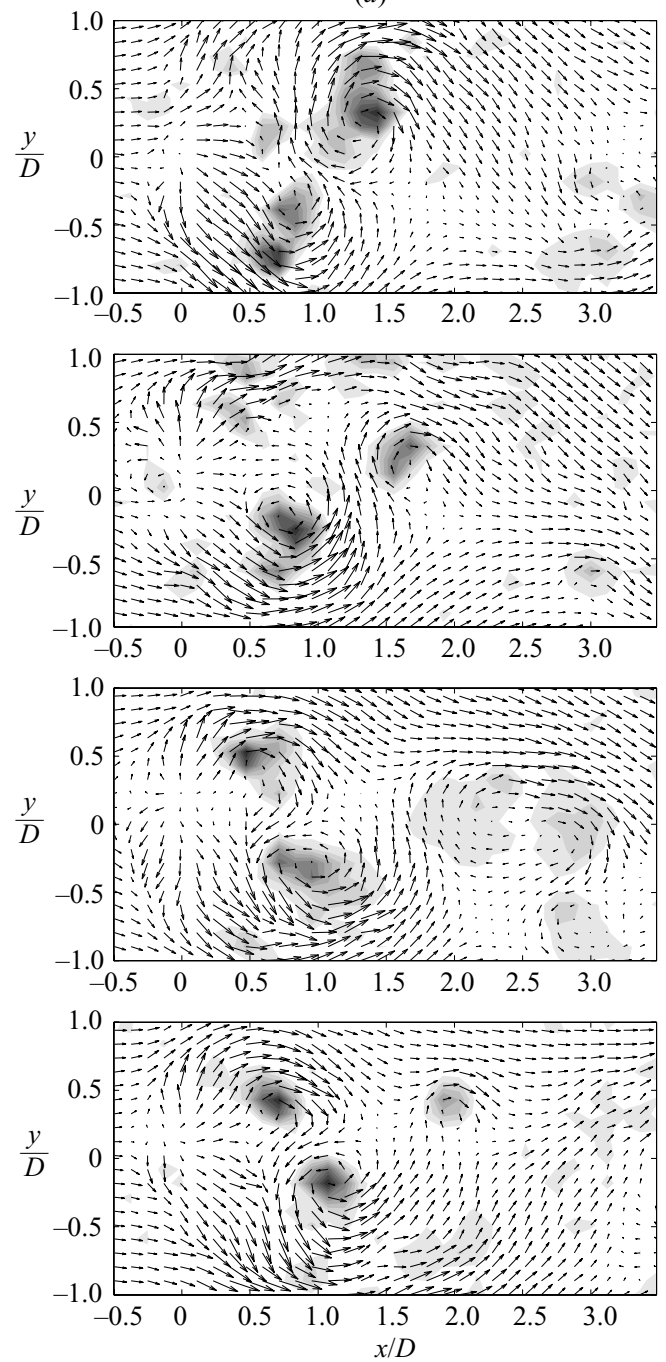

(b)


FIgURE 11. The modified $Q$-criterion used on snapshots $(a)$ and reconstructions $(b)$ of figure $10(z=1.33 D)$. Dark contours indicate positive values of $Q$.

downstream and a strong flow of crossflow fluid is found between this vortex and the jet core. The jet core is fairly symmetric and similar to the jet core seen in the mean velocity field (figure 6). In the jet core of the last snapshot (row 3) there is a flow for $y>0$ with a direction of $45^{\circ}$ to the $x$-axis. This flow interacts with the crossflow fluid and a new wake vortex appears at the downstream edge of the jet core. The dynamics of wake vortices in the $z=0.67 D$ plane is similar to those described in the previous section for the $z=1.33 \mathrm{D}$ plane. However, for the $z=0.67 \mathrm{D}$ plane, the variations in the jet core are significantly smaller and there is a much stronger interaction with the crossflow fluid coming into the wake.

The $Q$-criterion shown in figure 14 identifies all the wake vortices discussed above, but also reveals two additional vortices. The first is located near $(x / D, y / D)=$ $(0.4,0.5)$. It can be identified by the $Q$-criterion on most snapshots and also on the mean field (not shown). We find that this is the hanging vortex since it has mean flow 
(a)

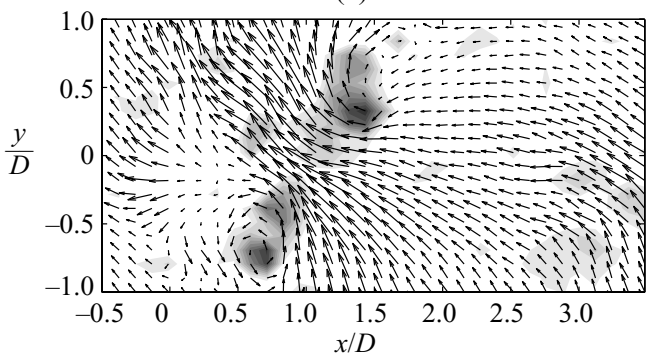

(b)



FIGURE 12. Galilean transformation of $(a)$ the upper plot from figure $11(a)(z=1.33 D)$ and (b) the upper plot from figure $14(a)(z=0.67 D$ - presented in $\S 5.2)$, respectively.

(a)
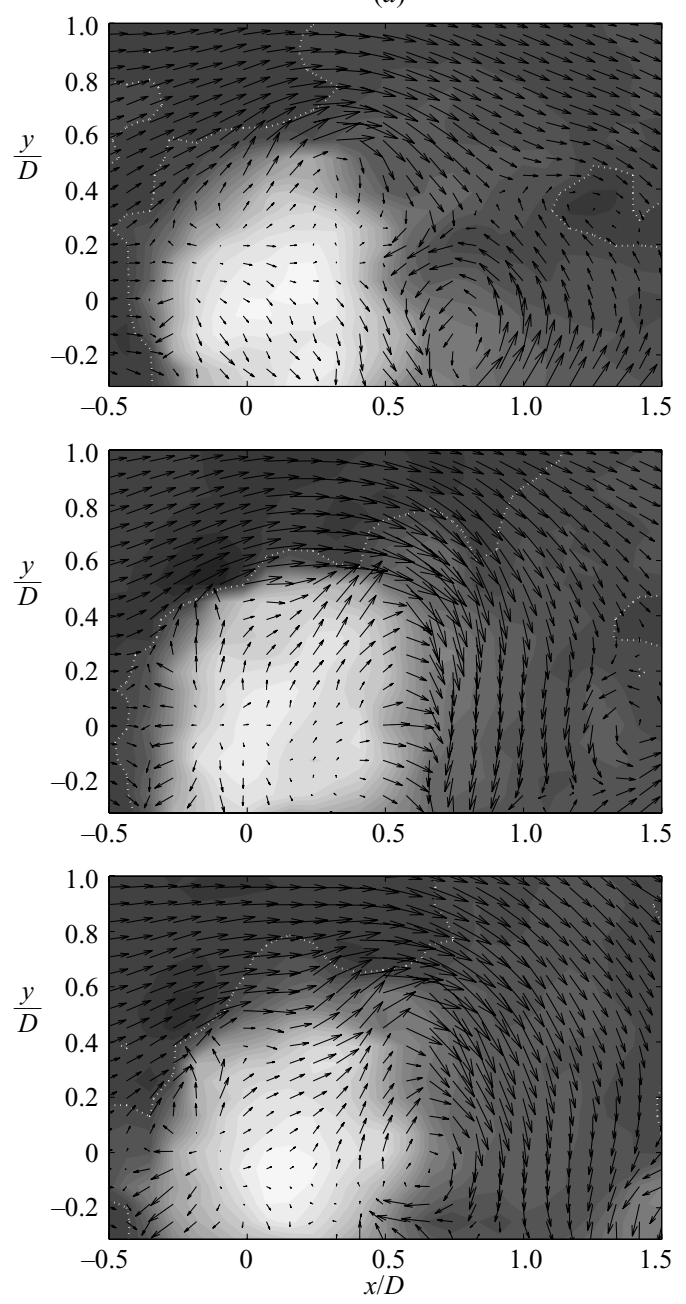

(b)
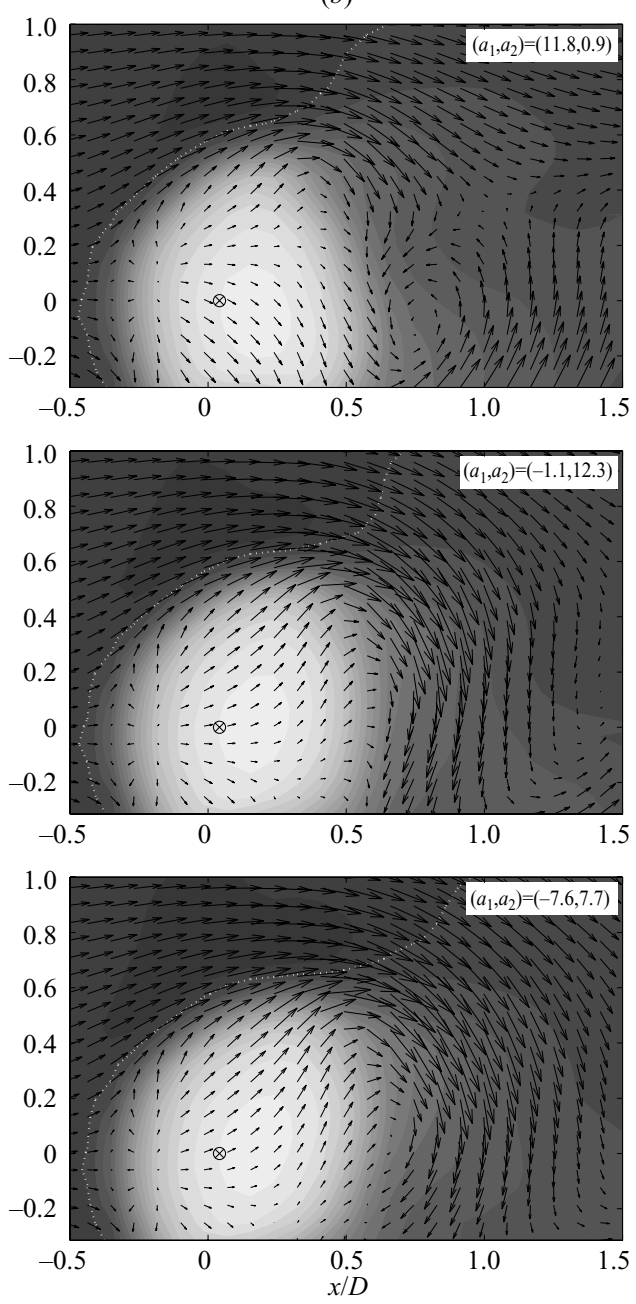

FIGURE 13. Snapshots from the $z=0.67 D$ plane $(a)$ and reconstructions $(b)$ using the first two POD modes 
(a)
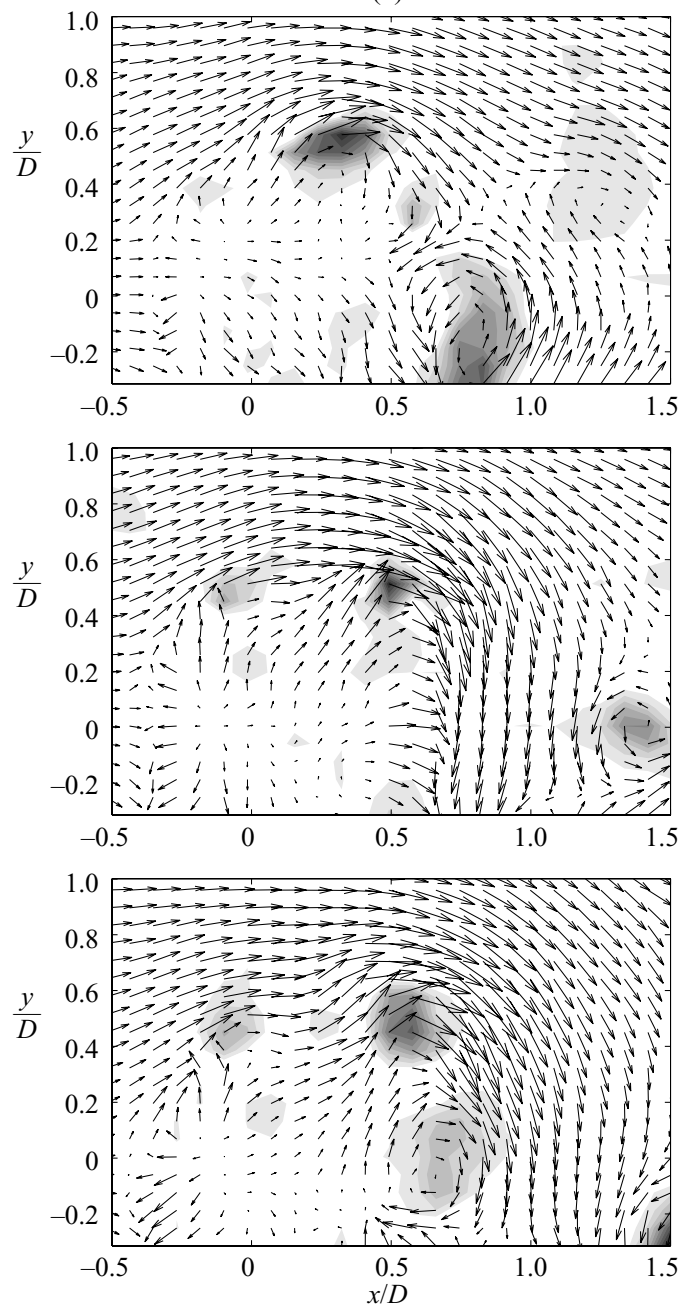

(b)
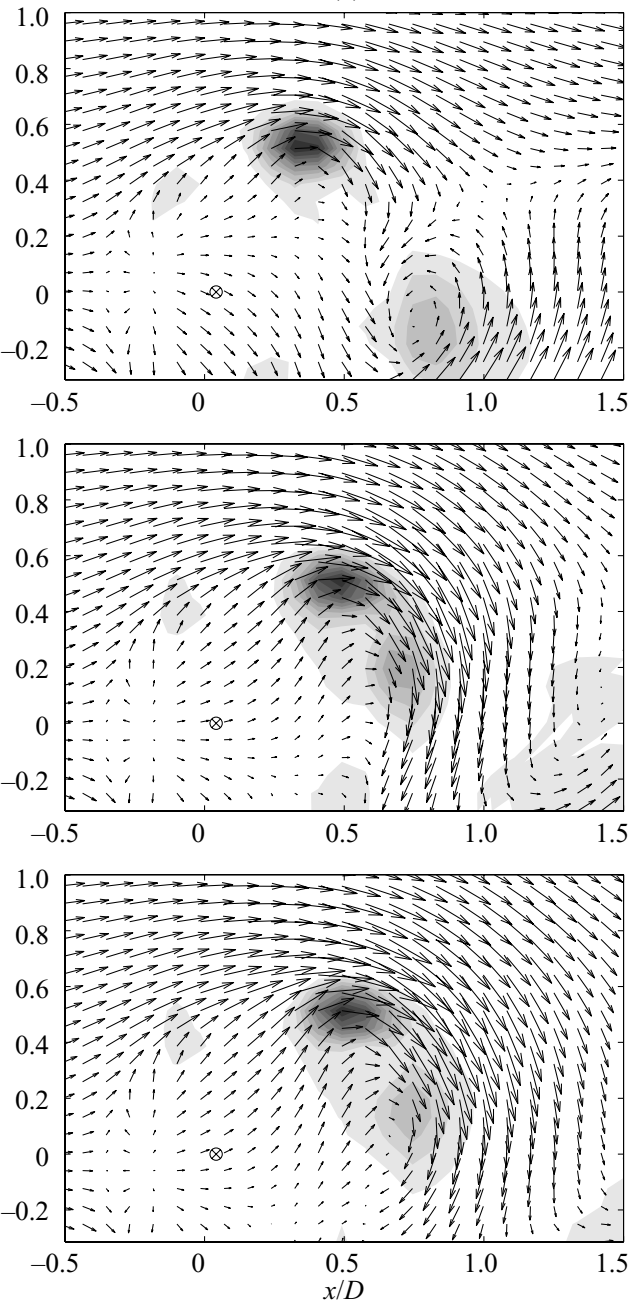

FIGURE 14. The modified $Q$-criterion used on snapshots $(a)$ and reconstructions $(b)$ of figure $13(z=0.67 D)$. Dark contours indicate positive values of $Q$.

definition and is found at the same position as described by Yuan et al. (1999). The hanging vortex is clearly separated from the wake vortex, both in snapshots and in reconstructions. The newly created wake vortex is seen in the reconstruction in row 2 of figure 14 even though it is not seen in the corresponding snapshot. This indicates that the creation typically happens at this point. A small vortex is identified in the two last snapshots (rows $2-3)$ in figure 14 at $(x / D, y / D)=(-0.1,0.5)$. In the first snapshot (row 1), a weak positive contour is seen at the same position. This vortex is also identified on many other snapshots (not shown) and a weak positive contour of $Q$ appears in the mean field and thus in the reconstructions shown in figure 14 . This vortex could be a part of a shear-layer vortex formed close to the wall. At some distance from the symmetry plane at $y=0$, the shear-layer vortices are bent in the jet direction to form side arms and will therefore give a contribution to the $z$-component 
(a)

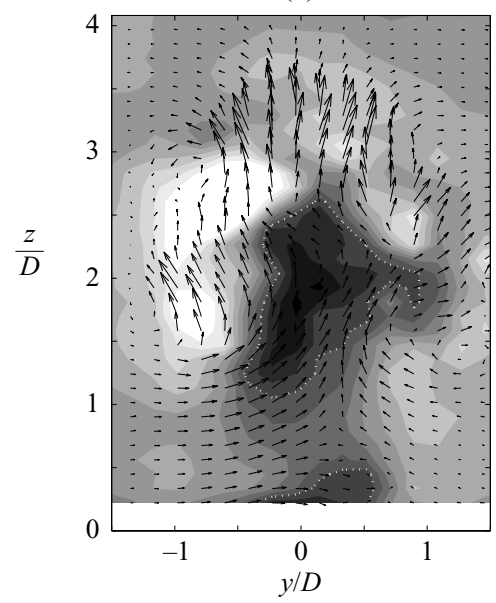

(b)

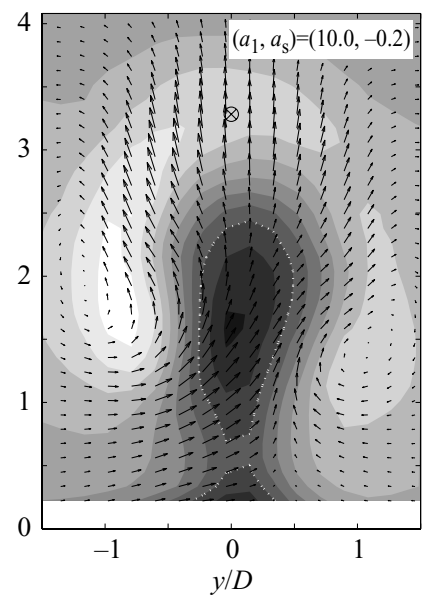

FIGURE 15. Snapshot from the $x=D$ plane $(a)$ and reconstruction $(b)$ using the first two POD modes. Maximum out-of-plane velocity in snapshot is $3.0 U_{\infty}$.

of the vorticity vector. The modified $Q$-criterion is not able to identify the shear-layer vortices close to $y=0$ since the out-of-plane gradients are not included.

\subsection{Snapshots at $x=D$ and $y=0$ planes}

Figure 15 shows a selected snapshot at the $x=D$ plane together with the corresponding reconstruction. The snapshot has been selected to have a dominating POD mode 1. This cut through the flow is more difficult to interpret than the two $z$-constant planes because the shear-layer vortices appear in the plane and because the axes of wake vortices form an angle to the plane. Concentrating on the wake vortex, the vortex centre seems to intersect the plane at about $(y / D, z / D)=(-0.5,2.0)$, since this is where there is a change of sign of the out-of-plane velocity and almost zero velocity component in the $y$-direction. Moving towards the edge of the wake (in the negative $y$-direction), a region near $(y / D, z / D)=(-1,2)$ with very high out-of-plane velocities (up to $3 U_{\infty}$ ) is found. Comparing with figure 10 , this flow must be jet fluid that is being deflected more than average and then is moving into the vortex. For lower values of $z$, the front of the vortex is seen. Here, fluid from the crossflow moves into the wake and is then accelerated upwards into the vortex. The vortex therefore seems to feed on fluid from the crossflow near the wall as suggested in figure 13 and on fluid from the jet at higher positions as suggested in figure 10 .

As discussed in $\S 4.4$, the CVP is being displaced by the passage of the wake vortex. The CVP is not easily identified on the snapshot. However with the help of the reconstruction that gives a more clean picture of the velocity field, it might be inferred based on in-plane vectors that the legs of the CVP are found near $(y / D, z / D)=(-1,1.6)$ and $(y / D, z / D)=(1,1.3)$. As discussed in $\S 4.4$, the actual centres of the CVP are probably closer to the symmetry plane $y=0$. However, the snapshot can still be taken as an indication that the leg of the CVP at negative $y$ has moved up while the leg at positive $y$ has moved down. For the $z=1.33 D$ plane it was found in $\S 5.1$ that the hanging vortex and the wake vortices might sometimes be merging. In figure 11 there are indications that this can happen near $x / D=1$. Such events would make it more difficult to distinguish between the hanging vortex/CVP and the wake vortices in the $x=D$ plane. 
(a)

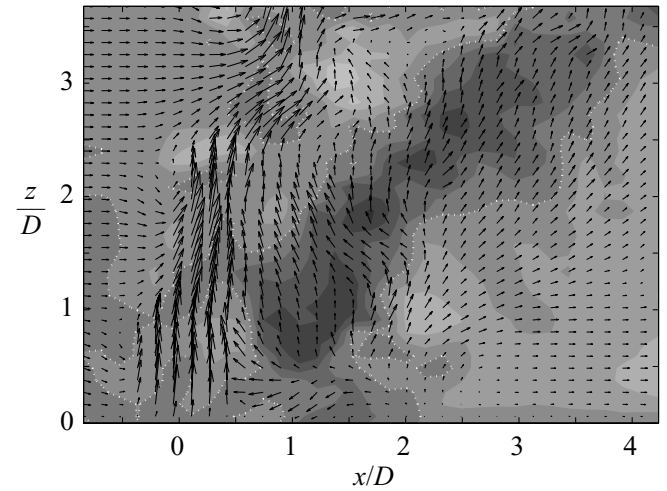

(b)

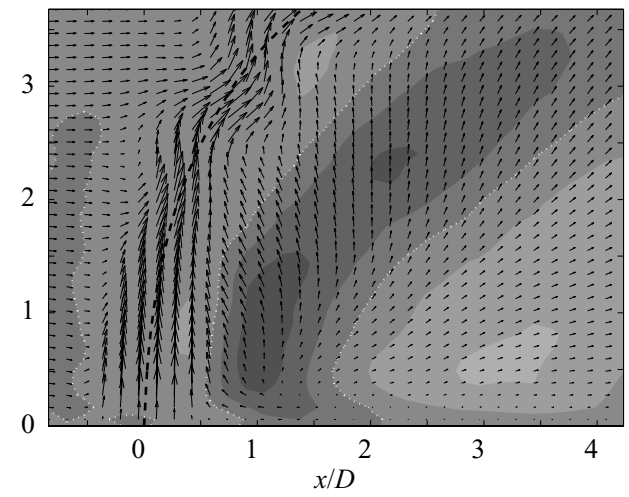

FIGURE 16. Snapshot from the $y=0$ plane $(a)$ and reconstruction $(b)$ using the first five POD modes and corresponding POD coefficients: $\left(a_{1}, a_{2}, a_{3}, a_{4}, a_{5}\right)=(9.5,-0.4,3.8,-3.1,5.5)$.

A short data series taken at the $x=2 D$ plane (not shown) had a mean field and first POD modes similar to those of the data in figure 7. Snapshots were also similar to the snapshot shown in figure 15. Comparison with Meyer et al. (2002b) (measured in the same flow facility with the same flow conditions) shows that the structure seen is indeed the CVP.

To complete the picture, a selected snapshot from the $y=0$ plane and the corresponding reconstruction are shown in figure 16. Again, the snapshot is selected to have a dominating POD mode 1 . In the reconstruction, the first five POD modes have been used in order to also reconstruct the shear-layer vortices. The out-of-plane velocity in the wake is in good overall agreement with the reconstruction, but with significant local variations. The in-plane velocities in the jet are dominated by the jet shear-layer vortices. These vortices move in the jet direction and the velocity vectors therefore do not form circular patterns unless e.g. the mean velocity field is subtracted.

\section{Further discussion of vortical structures}

The hanging vortex is seen on the snapshots in figure 14 as a strong vortex at the side of the jet. The strength and position varies, but it clearly has mean flow definition. Yuan et al. (1999) identify hanging vortices and argue that they are related to the skewed mixing layer on the sides of the jet. Kelso et al. (1996) also report vortex tubes originating at the sides of the jet and possibly contributing to the CVP. Yuan et al. (1999) find that the hanging vortex is a quasi-steady structure close to the wall, but at higher values of $z$, it behaves in an unpredictable manner: sometimes it sheds into the main flow and sometimes it will shed in the vertical direction. Their results suggest that the angle with the $z$-axis is roughly $30^{\circ}$ for $R \approx 3.3$. Margason (1993) presents static pressure contours on the flat plate and reports extreme values of the negative pressure coefficient around the two sides of the jet exit. We believe that the locations of minimum pressure correspond to the cores of the steady hanging vortices whose axes intersect the jet wall at around the same locations. Other vortical structures around these locations (such as the wake vortices) are highly unsteady and, therefore, not expected to leave a well-defined signature on the mean pressure field. Margason (1993) reports that the minimum value of the wall pressure coefficient is a function of $R$ and appears to reach its lowest value of -4.7 at $R=6$ and also that the location of the pressure minimum moves upstream from about $\phi=110^{\circ}$ for 
$R=2.2$ to $75^{\circ}$ for $R=10$ where $\phi$ is measured from the upstream edge of the jet exit. Therefore, we can assume that the hanging vortices are located around $\phi= \pm 90^{\circ}$ and move slightly upstream with increasing $R$. This starting position and angle agrees well with our observations at the $z=0.67 D$ and $z=1.33 D$ planes.

Both Kelso et al. (1996) and Yuan et al. (1999) find that the hanging vortex can break down at about the position where its axis goes into the wake of the jet. In Meyer et al. (2002b), the highest levels of turbulence kinetic energy in a mapping of the region near the jet exit of the present flow are found in two small regions near $(x / D, y / D, z / D)=(1.0, \pm 0.5,1.0)$, which is exactly where the hanging vortex axis goes into the jet wake. This all indicates that the hanging vortex is a part of important flow processes taking place in this region.

Based on observations from POD modes and snapshots, we now suggest a model for the wake vortices. The two first POD modes at the $y=0$ plane (figure 4) show interaction with the jet core in the region $0.5 \leqslant z / D<2$. Mode 1 has variations deepest into the jet core and a variation in out-of-plane velocity that probably shows the most upstream position of the wake vortices. We will therefore first focus on the flow described by the first POD mode in the $y=0$ plane. As noted earlier, this mode matches reasonably well with the first POD mode in the $x=D$ and $z=0.67 D$ planes and the second POD mode in the $z=1.33 D$ plane. Thus the following snapshots and corresponding reconstructions can be assumed to show the same step in time of the development of a wake vortex: the third snapshot (row 3) in figure 10, the first snapshot (row 1) in figure 13 and the snapshots in figures 15 and 16.

In the $z=1.33 D$ plane (figure 5), mode 2 has the strongest interaction with the jet core (note that the jet core is bent somewhat into the downstream direction at this plane). The snapshots at constant $z$-planes (figure 10, row 3 and figure 13, row 1) both show a vortex near $(x / D, y / D)=(1.0,-0.2)$ that is about to separate from the jet core. However, the snapshot at $z=1.33 D$ has the clearest signs of the appearance of a new vortex for $y / D>1$. This is seen both in the snapshots and in the reconstructions that are dominated by the first POD mode. The corresponding plot with the $Q$-criterion (row 3 in figure 11) has a weak indication of two cores within the vortex. This indicates that we might see both the hanging vortex and a newly created vortex here. In the corresponding plot (row 1) in figure 14, the hanging vortex and the newly created wake vortex are seen as clearly separate vortices. In summary, this indicates that the wake vortices are initiated at the downstream side of the jet core near $z / D \approx 1$. This is the location where the hanging vortices are assumed to 'break down'. It is therefore possible that the hanging vortices play a part in the initiation of the wake vortices. The wake vortex seems to entrain some jet fluid that is bent and turned into the wake. Both the first POD mode for the $y=0$ plane and the rest of the snapshots/reconstructions in figures 13-16 indicate that the vortex is quickly extended to cover the region from the wall to $z \approx 2 D$ where it meets the jet core. The first POD mode for the $y=0$ plane and the positions of the almost free vortex in figures 10 and 13 indicate that a curve through the centre of the vortex is almost parallel to the jet trajectory.

A vortex that is almost separated from the jet core is also seen on all snapshots/reconstructions that correspond to mode 1 in the $y=0$ plane. The snapshot at the $x=D$ plane (figure 15) indicates that the vortex has a tornadolike form and that it feeds mainly on fluid from the crossflow boundary layer at positions for $z / D<1$. It also receives jet fluid for $z / D>1$. This was also indicated by snapshots from the constant $z$-planes. Snapshots and reconstructions at the $x=D$ plane also show that the CVP has systematic fluctuations where one leg moves up and 


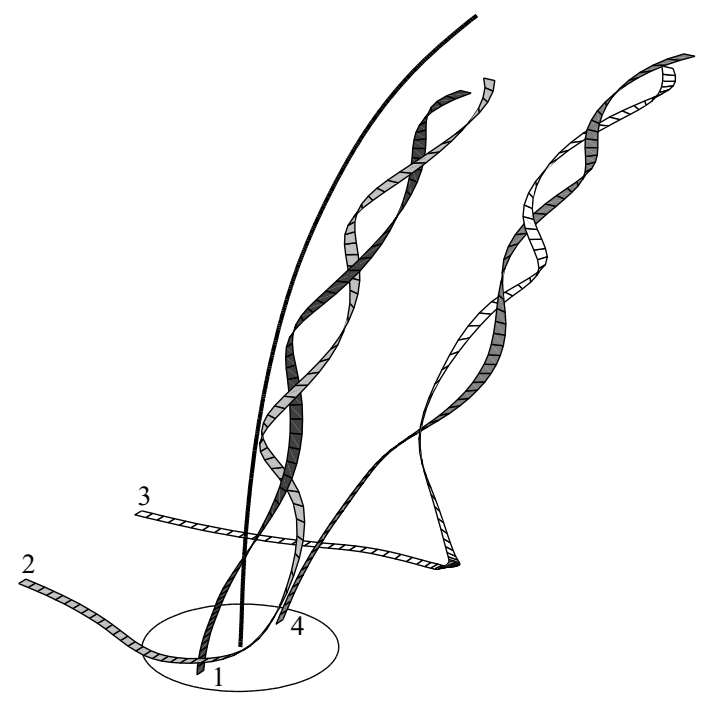

FiguRE 17. Sketch of model for the wake vortices. Traces of jet fluid ( 1 and 4$)$ and crossflow fluid ( 2 and 3 ) are illustrated by 'ribbons'. The solid line is the jet trajectory.

the other leg moves down during the passage of a wake vortex. This phenomenon has also been observed by Rivero et al. (2001). As already discussed, the creation of a wake vortex involves strong variations in the jet core near the jet exit. This is therefore the likely cause of the downstream fluctuations in the CVP.

The suggested model for the wake vortices can be summarized using figure 17 that shows a sketch of their formation. Idealized traces of jet and crossflow fluid, respectively, are shown as 'ribbons'. The ribbon marked 1 starts in the jet near one of the sides. The jet fluid at this point is deflected more than the jet trajectory and, at a height of about one diameter, it is moving into the wake where it is entrained by a wake vortex. This vortex will suck in wake fluid from the crossflow. The ribbon marked 2 shows how crossflow fluid is moved around the jet by potential flow forces and then sucked into the low-pressure wake region where it then meets the vortex entraining ribbon 1 . Both ribbons 1 and 2 come from regions with strong vorticity. This vorticity is being convected and turned to form the wake vortex. The ribbons marked 3 and 4 show how crossflow fluid and jet fluid, respectively, continues to feed a wake vortex at a position further downstream.

Fric \& Roshko (1994) and Kelso et al. (1996) visualized the wake vortices by placing a tracer in the crossflow boundary layer for a velocity ratio $(R=4$ and $\operatorname{Re}=3800)$ similar to those of the present study. As noted before, these studies involved laminar inflow conditions, which may create a flow different from the present one. Fric \& Roshko (1994) found that they could only visualize the vortex when the tracer was released in the incoming boundary layer and not when released in the jet. They concluded that the wake vortices are formed solely by fluid from the wall boundary layer. From the visualizations of both Fric \& Roshko (1994) and Kelso et al. (1996), we note two observations. The first is that the tracer released in the crossflow boundary layer shows vortical structures at $x \approx 0.5 D$ that are connected to the wall and have axes parallel to the $z$-axis. These vortices seem to penetrate into the jet core. The second observation is that when a tracer is released into the jet it spreads to quite a wide area in the $z$-direction as the jet bends into the crossflow. It is difficult from the 
visualizations to state that the tracer does not spread into a part of the wake. Since the wake vortices have a tornado-like form, fluid and vorticity entering the vortex will be transported upwards. A visualization of the present flow is therefore likely to give patterns similar to those discussed above: the lower part of the wake vortices will only contain crossflow fluid while the upper part will contain a mixture of both crossflow and jet fluid.

An interesting difference between the flow of the present study and flows involving laminar inflow conditions is the angle of the wake vortices. The visualizations by Fric \& Roshko (1994) show that the wake vortices connect to the jet core with an angle of about $90^{\circ}$ to the jet trajectory. The results in the present study on the other hand show vortices with axes that locally are parallel to the jet trajectory. This is an important difference that could be caused by the differences in inlet conditions. Another possibility is that the description of the wake vortices presented in the present paper is valid in the near field and that further downstream the wake vortices assume the shape described by Fric \& Roshko (1994). The wake vortices seem to be connected to the CVP and must therefore contribute to the CVP.

Results of Fric \& Roshko (1994) for $R \geqslant 2$ show that the wake vortices are shed into the flow in a periodic manner, whereas for $R<2$ Gopalan et al. (2004) report the presence of a semi-cylindrical vortical layer in the wake. In the latter case, it can be argued that a steady pair of tornado vortices is trapped in the vortical layer behind the jet. PIV measurements of Peterson \& Plesniak (2004) reveal steady wake vortices (called 'downstream spiral separation node' (DSSN) vortices) immediately downstream of jets emanating from short holes for $R=0.5$ and 1 . Peterson \& Plesniak (2004) state that the size of the DSSN vortices decreases with increasing $R$. Flow visualization results of Krothapalli, Lourenco \& Buchlin (1990) for a lowaspect-ratio rectangular jet at $R=3$ reveal a pair of strong and large steady vortices in the wake. The laser Doppler anemometry measurements and computational data of Gustaffson \& Johansson (2003) for slanted jets at $R=0.8$ also show steady-state wake vortices forming on top of two foci immediately downstream of the jet. All these observations agree with our measurements at $R=1.3$ where the POD analysis shows that moving wake vortices is not a typical pattern. By utilizing the pressure data presented in Margason (1993), we have already pointed out that the axes of the hanging vortices move in the downstream direction with decreasing velocity ratio. Therefore, we believe that the DSSN vortices are limiting forms of the hanging vortices for small velocity ratios.

Finally, we discuss the ideas existing in the literature on the formation of the CVP. Broadwell \& Breidenthal (1984) propose that the CVP is a result of the momentum of the jet, and that CVP formation is similar to that of wingtip vortices. This argument views the jet as a point source and does not address the actual vortex interactions that eventually generate the CVP. Fearn \& Weston (1974) argue that the CVP is formed at the jet exit as concentrated vortices. Coelho \& Hunt (1989) propose that the CVP is initiated within the pipe. Kelso et al. (1996) propose that the separation seen inside the pipe can be related to the CVP roll-up and that the shear-layer vortices fold and contribute to the circulation of the CVP. Muppidi \& Mahesh (2006) show that the pipe is not required to form the CVP, and that even a two-dimensional jet subject to a lateral acceleration gives rise to a CVP. Wee, Marzouk \& Ghoniem (2005) and Marzouk \& Ghoniem (2007) propose a mechanism by which the shear-layer vortices stretch and deform, and initiate the arms of the CVP, with the vorticity now aligned with the jet trajectory. In our view, the hanging vortices (DSSN vortices for low $R$ ) may be considered as the origin of the CVP. The hanging vortices have mean flow 
definition sufficiently close to the wall of the jet and they break down in a region where we have observed interaction with the wake vortices. Still, a significant part of the vorticity in the hanging vortices will be transported into the CVP. The CVP also receives contributions from the jet shear-layer vortices and the wake vortices, but this happens through non-stationary processes and the vorticity vector is aligned with the axis of the CVP at a more downstream (in the jet direction) position. According to our view, the CVP is formed at the jet exit (in the form of the hanging vortices) and the flow separation in the pipe only has a secondary effect on the formation. The contribution of the jet shear vortices to the CVP becomes apparent sufficiently far away from the wall as indicated by the vortex model computations of Wee et al. (2005), Marzouk \& Ghoniem (2007) and Cortelezzi \& Karagozian (2001).

\section{Conclusions}

The POD analyses of snapshots taken at different planes for $R=3.3$ all find that the first two modes represent significantly more energy than the following modes. The two modes can be interpreted as different phases in time of the same phenomenon. The modes from the different planes fit together qualitatively along the intersecting lines of the planes. For planes that intersect the jet core, the modes involve flow motions both in the jet core and in the wake. The flow phenomena captured by the modes can consistently be interpreted as wake vortices. Using this interpretation, the wakes vortices cause a strong interaction between the jet core and the crossflow.

The planes that intersect the upper part of the jet core seem to capture the shearlayer vortices in POD mode 3 and in following modes. Modes 1 and 2 have no activity in the region of the shear-layer vortices and the modes related to the shearlayer vortices have almost no activity in the region of the wake vortices. This is a strong indication that the two flow phenomena are not coupled.

The hanging vortices are identified in the measurements. The upper part of the hanging vortices seems to interact and at times merge with the newly formed wake vortices. However, we find that the hanging vortex is the first and most steady contributor to the formation of the CVP and that it therefore should be considered as the origin of the CVP.

A POD analysis for $R=1.3$ in the $y=0$ plane indicates that the wake vortices are a much less dominant flow feature at lower velocity ratios. Here the most dominant dynamical flow structure is the shear-layer vortices. Comparison of the data in the literature shows that the hanging vortices seem to move downstream to form two steady wake vortices right behind the jet core.

The above conclusions are based on data obtained for sufficiently high Reynolds numbers (turbulent inflow conditions) at two values of $R$, focusing on the $R=3.3$ case. In particular, the possibility of the secondary CVP causing the jet fluid in the wake at high $R$ and effect of various instability mechanisms on the flow structure at low Reynolds numbers may be the subjects of future studies.

The POD analysis of PIV snapshots has proven to be a powerful method of analysing dynamic flow features. It is also useful as a tool to interpret snapshots in order to distinguish between random flow variations and flow structures that appear with statistical weight. It is likely that this type of analysis can be a valuable aid in comparing numerical calculations, in particular large-eddy simulations (LES), to experimental results. The comparison of dynamical flow features could be an important supplement to the traditional comparison of time-averaged statistics. 
O.Ö. acknowledges a grant in scope of the NATO Science Fellowship Programme by the Scientific and Technical Research Council of Turkey and also the financial support of Department of Mechanical Engineering at DTU. We thank Professor Poul Scheel Larsen for his invaluable comments and suggestions.

\section{REFERENCES}

Adrian, R. J., Christensen, K. T. \& LiU, Z.-C. 2000 Analysis and interpretation of instantaneous turbulent fields. Exps. Fluids 29, 275-290.

Bernero, S. \& Fieldler, H. E. 2000 Application of particle image velocimetry and proper orthogonal decomposition to the study of a jet in counterflow. Exps. Fluids 29, S274-S281.

Blanchard, J. N., Brunet, Y. \& Merlen, A. 1999 Influence of a counter rotating vortex pair on the stability of a jet in a cross flow: an experimental study by flow visualizations. Exps. Fluids 26, 63-74.

Broadwell, J. \& Breidenthal, R. 1984 Structure and mixing of a transverse jet in incompressible flow. J. Fluid Mech. 148, 405-412.

Camussi, R., Guj, G. \& Stella, A. 2002 Experimental study of a jet in a crossflow at very low reynolds number. J. Fluid Mech. 454, 113-144.

Coelho, S. \& Hunt, J. 1989 The dynamics of the near field of strong jets in crossflows. J. Fluid Mech. 200, 95-120.

Cortelezzi, L. \& Karagozian, A. R. 2001 On the formation of the counter-rotating vortex pair in transverse jets. J. Fluid Mech. 446, 347-374.

FEARn, R. \& Weston, R. P. 1974 Vorticity associated with a jet in a cross flow. AIAA J. 12 (12), $1666-1671$.

FrIC, T. F. \& Roshko, A. 1994 Vortical structure in the wake of a transverse jet. J. Fluid Mech. 279, $1-47$.

Fukunaga, K. 1990 Introduction to Statistical Pattern Recognition, 2nd edn. Academic Press.

Gopalan, S., Abraham, B. M. \& Katz, J. 2004 The structure of a jet in cross flow at low velocity ratios. Phys. Fluids 16 (6), 2067-2087.

Graftieaux, L., Michard, M. \& Grosjean, N. 2001 Combining PIV, POD and vortex identification algorithms for the study of unsteady turbulent swirling flows. Meas. Sci. Technol. 12, 14221429.

Gustaffson, K. \& Johansson, T. 2003 Turbulence and velocity fields of slanted jets in crossflow- measurements and CFD simulations. In Turbulence, Heat and Mass Transfer 4 (ed. K. Hanjalic, Y. Nagano \& M. Tummers). Antalya, Turkey.

Hasselbrink, E. F. \& Mungal, M. G. 2001 Transverse jets and jet flames. Part 2. Velocity and OH field imaging. J. Fluid Mech. 443, 27-68.

Haven, B. \& Kurosaka, M. 1997 Kidney and anti-kidney vortices in crossflow jets. J. Fluid Mech. 352, 27-64.

Holmes, P., Lumley, J. L. \& Berkooz, G. 1998 Turbulence, Coherent Structures, Dynamical Systems and Symmetry. Cambridge University Press.

Hunt, J., Wray, A. \& Moin, P. 1988 Eddies, stream and convergence zones in turbulent flows. Tech. Rep. Center for Turbulence Research, NASA-Ames Research Center and Stanford University, California, USA.

Kelso, R. M., Lim, T. T. \& Perry, A. E. 1996 An experimental study of round jets in cross flow. J. Fluid Mech. 306, 111-144.

Krothapalli, A., Lourenco, L. \& Buchlin, J. 1990 Separated flow upstream of a jet in crossflow. AIAA J. 28, 414-420.

Lim, T. T., New, T. H. \& LuO, S. C. 2001 On the development of large-scale structures of a jet normal to a cross flow. Phys. Fluids 13 (3), 770-775.

Lumley, J. L. 1967 The structure of inhomogeneous turbulent flow. In Atmospheric Turbulence and Radio Wave Propagation (ed. A. M. Yaglom \& V. I. Tatarski), pp. 166-178, Nauka, Moscow.

Margason, R. J. 1993 Fifty years of jet in crossflow research. In Computational and Experimental Assessment of Jets in Cross Flow. AGARD-CP-534, Winchester, UK.

Marzouk, Y. M. \& Ghoniem, A. F. 2007 Vorticity structure and evolution in a transverse jet. J. Fluid Mech. 575, 267-305. 
Meyer, K. E., Özcan, O., Larsen, P. S., Gjelstrup, P. \& Westergaard, C. H. $2002 a$ Point and planer lif for velocity-concentration correlations in a jet in crossflow. In Laser Techniques for Fluid Mechanics (ed. R. J. Adrian et al.). Springer.

Meyer, K. E., Özcan, O. \& WestergaARd, C. H. $2002 b$ Flow mapping of a jet in crossflow with stereoscopic PIV. J. Visualization 5 (3), 225-231.

Moussa, Z., TrischKa, J. \& EskinazI, S. 1977 The near field in the mixing of a round jet with a cross-stream. J. Fluid Mech. 80, 49-80.

MuPPIDI, S. \& MAHESH, K. 2005 Study of trajectories in crossflow using direct numerical simulations. J. Fluid Mech. 530, 81-100.

MupPIDI, S. \& MAHESH, K. 2006 Two-dimensional model problem to explain counter-rotating vortex pair formation in a transverse jet. Phys. Fluids 18 (8), 085103.

New, T. H., Lim, T. T. \& Luo, S. C. 2004 A flow field study of an elliptic jet in cross flow using DPIV technique. Exps. Fluids 36 (4), 604-618.

ÖZCAN, O. \& LARSEN, P. S. 2001 An experimental study of a turbulent jet in cross-flow by using LDA. Tech. Rep. MEK-FM 2001-02. Department of Mechanical Engineering, Technical University of Denmark.

ÖZCAN, O. \& LARSEN, P. S. 2003 Laser doppler anemometry study of a turbulent jet in crossflow. AIAA J. 41 (8), 1614-1615.

Özcan, O., Meyer, K. E. \& Larsen, P. S. 2005 Measurement of mean rotation and strain-rate tensors by using stereoscopic PIV. Exps. Fluids 39 (4), 771-783.

Pedersen, J. M. 2003 Analysis of planar measurements of turbulent flows. PhD thesis, Department of Mechanical Engineering, Technical University of Denmark.

Pedersen, J. M. \& Meyer, K. E. 2002 POD-analysis of flow structures in a scale model of a ventilated room. Exps. Fluids 33 (6), 940-949.

Peterson, S. D. \& Plesniak, M. W. 2004 Evolution of jets imanating from short holes into crossflow. J. Fluid Mech. 503, 57-91.

Rivero, A., Ferré, J. A. \& Giralt, F. 2001 Organized motions in a jet in crossflow. J. Fluid Mech. 444, 117-149.

Shapiro, S. R., King, J. M., MCloskey, R. T. \& Karagozian, A. R. 2006 Optimization of controlled jets in crossflow. AIAA J. 44, 1292-1298.

Sirovich, L. 1987 Turbulence and the dynamics of coherent structures. Part I: Coherent structures. Q. Appl. Maths. 45 (3), 561-571.

Smith, S. H. \& Mungal, M. G. 1998 Mixing, structure and scaling of the jet in crossflow. J. Fluid Mech. 357, 83-122.

SpalarT, P. R. 1988 Direct simulation of a turbulent boundary layer up to $R_{\theta}=1410$. J. Fluid Mech. 187, 61-98.

Su, L. K. \& Mungal, M. G. 2004 Simultaneous measurements of scalar and velocity field evolution in turbulent crossflowing jets. J. Fluid Mech. 513, 1-45.

WeE, D., Marzouk, Y. M. \& Ghoniem, A. F. 2005 Lagrangian simulation of a jet-in-crossflow at a finite reynolds number. AIAA Paper 2005-0291.

Yuan, L. L., Street, R. L. \& Ferziger, J. H. 1999 Large-eddy simulations of a round jet in crossflow. J. Fluid Mech. 379, 71-104. 